\title{
Fooling Gaussian PTFs via Local Hyperconcentration
}

\author{
Ryan O’Donnell ${ }^{*}$ \\ odonnell@cs.cmu.edu \\ Carnegie Mellon University \\ Pittsburgh, Pennsylvania, U.S.A.
}

\author{
Rocco A. Servedio ${ }^{\dagger}$ \\ rocco@cs.columbia.edu \\ Columbia University \\ New York, New York, U.S.A.
}

\author{
Li-Yang $\operatorname{Tan}^{\ddagger}$ \\ liyang@cs.stanford.edu \\ Stanford University \\ Stanford, California, U.S.A.
}

\begin{abstract}
We give a pseudorandom generator that fools degree- $d$ polynomial threshold functions over $n$-dimensional Gaussian space with seed length $d^{O(\log d)} \cdot \log n$. All previous generators had a seed length with at least a $2^{d}$ dependence on $d$.

The key new ingredient is our Local Hyperconcentration Theorem, which shows that every degree- $d$ Gaussian polynomial is hyperconcentrated almost everywhere at scale $d^{-O(\log d)}$.
\end{abstract}

\section{CCS CONCEPTS}

- Theory of computation $\rightarrow$ Pseudorandomness and derandomization.

\section{KEYWORDS}

pseudorandomness, polynomial threshold functions, Gaussian space

\section{ACM Reference Format:}

Ryan O'Donnell, Rocco A. Servedio, and Li-Yang Tan. 2020. Fooling Gaussian

PTFs via Local Hyperconcentration. In Proceedings of the 52nd Annual ACM SIGACT Symposium on Theory of Computing (STOC '20), June 22-26, 2020, Chicago, IL, USA. ACM, New York, NY, USA, 14 pages. https://doi.org/10. $1145 / 3357713.3384281$

\section{INTRODUCTION}

This paper is about pseudorandom generators (PRGs) for polynomial threshold functions (PTFs) over Gaussian space. Let us explain what this means. Let $\mathscr{C}$ be a class of functions from $\mathbb{R}^{n}$ to $\mathbb{R}$. A distribution $\mathcal{G}$ over $\mathbb{R}^{n}$ is an $\varepsilon$-PRG for $\mathscr{C}$ over Gaussian space if for every function $f \in \mathscr{C}$,

$$
\left|\underset{\boldsymbol{z} \sim \mathcal{G}}{\mathbf{E}}[f(\boldsymbol{z})]-\underset{\boldsymbol{x} \sim \mathcal{N}(0,1)^{n}}{\mathbf{E}}[f(\boldsymbol{x})]\right| \leq \varepsilon,
$$

where $\mathcal{N}(0,1)^{n}$ is the standard $n$-dimensional Gaussian distribution. We equivalently say that $\mathcal{G} \varepsilon$-fools $\mathscr{C}$ over Gaussian space. If a draw

\footnotetext{
*Supported by NSF grant CCF-1717606.

${ }^{\dagger}$ Supported by NSF grants CCF-1814873, IIS-1838154, CCF-1563155, and by the Simons Collaboration on Algorithms and Geometry.

¥Supported by NSF grant CCF-192179 and NSF CARERR Award CCF-1942123.

Permission to make digital or hard copies of all or part of this work for personal or classroom use is granted without fee provided that copies are not made or distributed for profit or commercial advantage and that copies bear this notice and the full citation on the first page. Copyrights for components of this work owned by others than ACM must be honored. Abstracting with credit is permitted. To copy otherwise, or republish, to post on servers or to redistribute to lists, requires prior specific permission and/or a fee. Request permissions from permissions@acm.org.

STOC '20, June 22-26, 2020, Chicago, IL, USA

(C) 2020 Association for Computing Machinery.

ACM ISBN 978-1-4503-6979-4/20/06 . .\$15.00

https://doi.org/10.1145/3357713.3384281
}

$z \sim \mathcal{G}$ can be deterministically generated from a source of $s$ independent uniformly random bits, we say that the seed length of $z$ is $s$. If furthermore the generation can be performed by a computationally efficient algorithm, we say the PRG is explicit.

A degree-d polynomial threshold function (PTF) is a function $f(x)=\operatorname{sign}(p(x))$ where $p: \mathbb{R}^{n} \rightarrow \mathbb{R}$ is a real polynomial of total degree at most $d$. Now we can state our main theorem:

Theorem 1.1. For all $n, d \in \mathbb{N}$ and $\varepsilon \in(0,1)$, there is an explicit $P R G$ with seed length $(d / \varepsilon)^{O(\log d)} \cdot \log n$ that $\varepsilon$-fools the class of all degree-d PTFs over n-dimensional Gaussian space.

The quasipolynomial dependence on $d$ here is a substantial improvement over previous PRGs, all of which had at least $2^{O(d)}$ dependence or worse. We view this as notable, as there are few prior works concerning structural properties of $n$-dimensional Gaussian or Boolean PTFs that are nontrivial for $d \gg \log n$.

\subsection{Prior Work}

There has been significant work on PRGs for PTFs. Their study was initiated by Meka and Zuckerman $[9,10]$, who gave a PRG with seed length ${ }^{1}(d / \varepsilon)^{O(d)} \cdot \log n$ that fools degree- $d$ PTFs over the more general setting of Boolean space, $\{ \pm 1\}^{n}$. PRGs over Boolean space can be shown to also yield PRGs over Gaussian space, thanks to the fact that $\boldsymbol{x}_{1}+\cdots+\boldsymbol{x}_{m}$ has a nearly Gaussian distribution when $\boldsymbol{x} \sim\{ \pm 1\}^{m}$ is uniformly random (see the discussion in Section 3.1), and the fact that degree- $d$ PTFs are closed under taking linear combinations of inputs. Since the work of $[9,10]$, there have been several works that focus just on fooling PTFs over Gaussian space, which we now discuss.

First, Kane [3] showed that limited independence (see Definition 3.1) suffices to fool Gaussian PTFs. The amount of independence required was $O_{d}\left(\varepsilon^{-2^{O(d)}}\right)$, which translates into $O_{d}\left(\varepsilon^{-2^{O(d)}}\right)$. $\log n$ in seed length. Using a different generator (one that is not based only on limited independence), Kane [4] then gave a PRG for Gaussian PTFs with seed length $2^{O(d)} \cdot \operatorname{poly}(1 / \varepsilon) \cdot \log n$. Note that this seed length strictly improves upon that in [10], albeit only in the Gaussian setting.

Towards further improving the seed length dependence on $\varepsilon$, Kane [5] gave a PRG with seed length $A(d, 1 / c) \cdot(1 / \varepsilon)^{2+c} \cdot \log n$ for any $c>0$, where $A(\cdot, \cdot)$ is a variant of the Ackermann function. ${ }^{2}$ This was improved to $A(d, 1 / c) \cdot(1 / \varepsilon)^{c} \cdot \log n$ in [6]; while the seed length now has subpolynomial dependence on $1 / \varepsilon$, its dependence

\footnotetext{
${ }^{1}$ They state $O(1 / \varepsilon)^{O(d)} \cdot \log n$ just after [10, Thm. 5.18], but they have appear to have dropped a factor of $d$ when citing their Thm. 5.2 at the end of Lem. 5.20's proof. Correcting this leads to the seed length $(d / \varepsilon)^{O(d)} \cdot \log n$.

${ }^{2}$ In fact, it seems that correcting a typo in [3, Proof of Prop. 12], where a " $\theta$ " factor should be " $\theta^{2}$ ", already leads to seed length $2^{O_{c}(d)} \cdot(1 / \varepsilon)^{2+c} \cdot \log n$.
} 
Table 1: Our work and the prior results on fooling degree- $d$ Gaussian PTFs. The last column indicates the range of values of $d$ 's for which the seed length of the corresponding PRG is nontrivial (i.e. $o(n)$ ). The generators of [2, 10] work for the more general setting of Boolean space, and [2,3]'s analyses show that limited independence suffices.

\begin{tabular}{|c|l|l|}
\hline Reference & \multicolumn{1}{|c|}{ Seed length } & Allowable / nontrivial range of $d$ 's \\
\hline$[2]$ & $\tilde{O}\left(1 / \varepsilon^{9}\right) \cdot \log n$ & $d \leq 2$ \\
\hline$[9,10]$ & $(d / \varepsilon)^{O(d)} \cdot \log n$ & $d \leq O(\log n / \log \log n)$ \\
\hline$[3]$ & $O_{d}\left(\varepsilon^{-2^{O(d)}}\right) \cdot \log n$ & $d \leq$ slightly superconstant \\
\hline$[4]$ & $2^{O(d)} \cdot \operatorname{poly}(1 / \varepsilon) \cdot \log n$ & $d \leq O(\log n)$ \\
\hline$[5]$ & $A\left(d, \frac{1}{c}\right) \cdot(1 / \varepsilon)^{2+c} \cdot \log n$ for any $c>0$ & $d \leq$ slightly superconstant \\
\hline$[6]$ & $A\left(d, \frac{1}{c}\right) \cdot(1 / \varepsilon)^{c} \cdot \log n$ for any $c>0$ & $d \leq$ slightly superconstant \\
\hline$[7]$ & $O(\log 6(1 / \varepsilon) \log \log (n / \varepsilon) \log n)$ & $d \leq 2$ \\
\hline$[8]$ & $O(\log (1 / \varepsilon) \log \log (1 / \varepsilon)+\log n)$ & $d=1$ \\
\hline This work & $(d / \varepsilon)^{O(\log d)} \cdot \log n$ & $d \leq 2$ \\
\hline
\end{tabular}

on $d$ limits its applicability to PTFs of constant (or very slightly superconstant) degree.

For degree-2 PTFs, Kane [7] gives a PRG with seed length

$$
O\left(\log ^{6}(1 / \varepsilon) \log \log (n / \varepsilon) \log n\right) ;
$$

Diakonikolas, Kane, and Nelson [2] showed that $\tilde{O}\left(1 / \varepsilon^{9}\right)$-wise independence suffices to fool degree-2 PTFs over both Boolean and Gaussian space. For degree-1 PTFs (i.e. halfspaces), the current best PRG is due to Kothari and Meka who achieve a near-optimal seed length of $O(\log (1 / \varepsilon) \log \log (1 / \varepsilon)+\log n)$ [8].

Summarizing the prior state of the art, previous PRGs were either specific to $d=1,2$, or else had seed length with at least an exponential dependence on $d$. Consequently, there were no PRGs that could fool PTFs of degree $d=\log n$, even just to constant accuracy $\varepsilon$. Theorem 1.1 therefore represents the first PRG that is able to fool PTFs of degree $d \geq \log n$; our seed length remains nontrivial for $d$ as large as $2^{\Omega(\sqrt{\log n})}$. Please see Table 1 .

\subsection{Motivations}

Geometric Content. We now give a geometric perspective on the problem of constructing PRGs for Gaussian PTFs. Suppose one is given a set $F \subseteq \mathbb{R}^{n}$ and one wishes to approximately compute its Gaussian volume, $\operatorname{Pr}_{\boldsymbol{x} \sim \mathcal{N}(0,1)^{n}}[\boldsymbol{x} \in F]$. There is an obvious Monte Carlo approach: picking $O\left(1 / \varepsilon^{2}\right)$ Gaussian vectors $x$ at random and outputting the fraction that fall into $F$ will, with high probability, give an $\varepsilon$-accurate estimate. Our question is to what extent randomness is necessary for this problem.

The extent to which derandomization is possible depends on the "complexity" of the sets $F$ we allow. If $F$ is only given via a black-box membership oracle then no derandomization is possible. So we need to assume an "explicit description" of $F$ is given, and in this paper we focus on the case that $F$ is the set of points satisfying a polynomial inequality of degree at most $d$ (i.e., $F$ is the set indicated by a degree- $d$ PTF). Thus the $d=1$ case allows halfspaces, the $d=2$ case allows ellipsoids and hyperboloids, etc.

One natural approach to this volume-approximation problem is the following: First, define some kind of explicit (nonrandom) finite "grid" of discrete points in $\mathbb{R}^{n}$; second, show that the Gaussian volume of any degree- $d$ PTF set $F$ is closely approximated by the fraction of grid points in $F$. A naive gridding scheme would use at least an exponential-in- $n$ number of grid points (even for $d=$ $O(1)$ ); the question is whether we can use a subexponential-in- $n$ number of gridpoints, when $d \ll n$. Our Theorem 1.1 provides such a solution; by enumerating all seeds (essentially, taking the support of $\mathcal{G}$ ), we get an explicit set of just $n^{d^{O(\log d)}}$ "grid points" that gives a high-quality volume approximation for any degree$d$ polynomial threshold set; this is nontrivial for $d$ up to some $2^{\Theta(\sqrt{\log d})}$. Also note that this kind of "PRG solution" is stronger than just being an "volume-approximation" algorithm of the type "given $F$, approximate $\operatorname{vol}(F)$ "; as it is PRG-based, it gives one fixed, deterministic "grid" that simultaneously works to approximate the volume of all degree- $d$ polynomial threshold sets $F$.

Boolean Complexity Theory. As mentioned earlier, the problem of PRGs (or volume-approximation) for Gaussian polynomial threshold functions is a special case of the problem of PRGs (or approximatecounting) for Boolean polynomial threshold functions. This, in turn, is a very special case of the problem of derandomization for general Boolean circuits. Recall that the BPP vs. P problem is roughly equivalent to asking whether there is a deterministic polynomial-time algorithm that, given the explicit description of a subset $F \subseteq\{0,1\}^{n}$ in the form of a poly $(n)$-gate Boolean circuit $C$ computing the indicator function of $F$, computes a .1-accurate approximation to its "volume", $\operatorname{Pr}_{\boldsymbol{x} \sim\{0,1\}^{n}}[C(\boldsymbol{x})=1]$. Given how far we are from answering this question, the field of pseudorandomness has focused 
on special classes of circuits, of restricted depth and gate-types; the case of Boolean PTFs corresponds to depth- 2 circuits $C$ with a threshold gate on top and AND gates of width at most $d$ at the bottom.

\subsection{Our Key New Tool: The Local Hyperconcentration Theorem}

For large $d$, the best prior PRG for degree- $d$ Gaussian PTFs is Kane's [4], which has seed length $2^{O(d)} / \operatorname{poly}(\varepsilon) \cdot \log n$. In this section we describe the most important new ingredient we introduce to Kane's framework, which lets us reduce the seed length's dependence on $d$ down to $d^{O(\log d)}$. In the next section we will give an overview of the constructions of $[4,9,10]$, putting our new tool into context.

We call our main new tool the Local Hyperconcentration Theorem. To explain it, suppose $p: \mathbb{R}^{n} \rightarrow \mathbb{R}$ is a degree- $d$ polynomial. Since $p$ has high degree, it might fluctuate quite wildly near a given point $x \in \mathbb{R}^{n}$, causing $\operatorname{sign}(p(x))$ to rapidly switch between \pm 1 in small neighborhoods. However, we might hope that for most points $x$, the value of $p$ in a local neighborhood of $x$ is almost always within a $1 \pm \delta$ multiplicative factor of $p(x)$, and hence is almost always of constant sign.

The right definition of a "local neighborhood of $x$ " is to choose a small scale parameter $\lambda>0$, and then to consider a Gaussian $\widetilde{\boldsymbol{x}}$, centered at $\sqrt{1-\lambda} x$, with variance $\lambda$ in each coordinate. ${ }^{3}$ Now if $\operatorname{Var}[p(\widetilde{\boldsymbol{x}})] \ll \mathrm{E}[p(\widetilde{\boldsymbol{x}})]^{2}$, we may say that $p$ is (multiplicatively) concentrated in this $\lambda$-local neighborhood of $x$; and indeed, the second moment method (Chebyshev's inequality) tells us that $p(\widetilde{\boldsymbol{x}})$ almost always has the same sign (namely, the sign of $\mathbf{E}[p(\widetilde{\boldsymbol{x}})])$. The most important ingredient in Kane's work, [4, Cor. 10+Lem. 11], establishes this sort of result:

TheOREM 1.2 (KANE'S Key TECHNICAL THEOREM, SIMPLIFIED). Let $p: \mathbb{R}^{n} \rightarrow \mathbb{R}$ be a degree-d polynomial. Provided $\lambda \leq 2^{-O(d)}$, with high probability over $\boldsymbol{x} \sim \mathcal{N}(0,1)^{n}$ we have

$$
\operatorname{Var}[p(\widetilde{\boldsymbol{x}})] \ll \mathbf{E}[p(\widetilde{\boldsymbol{x}})]^{2}, \quad \text { where } \widetilde{\boldsymbol{x}} \sim \sqrt{1-\lambda} \boldsymbol{x}+\mathcal{N}(0, \lambda)^{n} .
$$

We may say that Kane shows degree- $d$ polynomials have local concentration at scale $\lambda=2^{-O(d)}$, almost everywhere. The value $L=1 / \lambda=2^{O(d)}$ ends up becoming the dominant factor in Kane's PRG's seed length. At a high level, this is because the PRG has the form $z=w_{1}+w_{2}+\cdots+w_{L}$, where the $\boldsymbol{w}_{i}$ 's are independent random vectors with $O(d)$-wise-independent $\mathcal{N}(0, \lambda)^{n}$ distributions.

By way of contrast, our new Local Hyperconcentration Theorem (stated in simplified form below) shows local hyperconcentration at scale $\lambda=d^{-O(\log d)}$. For a high level sketch of the proof, see Section 5.1 .

Theorem 1.3 (Simplified Local Hyperconcentration TheoREM, SEE TheOREM 5.1:). Let $p: \mathbb{R}^{n} \rightarrow \mathbb{R}$ be a degree-d polynomial. Provided $\lambda \leq d^{-O(\log d)}$, with high probability over $\boldsymbol{x} \sim \mathcal{N}(0,1)^{n}$ we

\footnotetext{
${ }^{3}$ The $\sqrt{1-\lambda}$ factor is included so that when we look at a typical $\boldsymbol{x}$ chosen from $\mathcal{N}(0,1)^{n}$, the resulting "random point in the neighborhood" $\widetilde{\boldsymbol{x}}$ also has distribution $\mathcal{N}(0,1)^{n}$
}

have

$$
\operatorname{HyperVar}_{R}[p(\widetilde{\boldsymbol{x}})] \ll \mathrm{E}[p(\widetilde{\boldsymbol{x}})]^{2} \text {, where } \widetilde{\boldsymbol{x}} \sim \sqrt{1-\lambda} \boldsymbol{x}+\mathcal{N}(0, \lambda)^{n},
$$

for any large constant $R$ (indeed, for any $\left.R \leq d^{O(\log d)}\right)$.

We will define "hypervariance" $\operatorname{HyperVar}_{R}[\cdot]$ later (see Definition 3.17); here we only note that it is a stronger notion than variance, in the sense that $\operatorname{HyperVar}_{R}[p]$ is always at least as large as $\operatorname{Var}[p]$ for all $R \geq 1$. Whenever the theorem's conclusion holds for an outcome $x$ of $\boldsymbol{x}$, the value of $p$ in the $\lambda$-local neighborhood of $x$ is "hyperconcentrated" (see Lemma 3.21), meaning that for any large constant $q$,

$$
\mathrm{E}\left[|p(\widetilde{\boldsymbol{x}})-\mu|^{q}\right] \ll|\mu|^{q}, \quad \text { where } \mu=\mathrm{E}[p(\widetilde{\boldsymbol{x}})] .
$$

The case $q=2$ here is precisely the "concentration" conclusion in Kane's theorem. Our hyperconcentration is a stronger conclusion: e.g., taking $q=4$ lets us use the "fourth moment method", and in fact we'll eventually use $q=8$.

To summarize, our theorem has two important improvements over Kane's. First, it shows concentration at a much larger scale, $\lambda=d^{-O(\log d)}$, rather than $2^{-O(d)}$. This crucially gives us the potential to get our seed's dependence on $d$ to be $1 / \lambda=d^{O(\log d)}$. This is far from automatic, though, because there are several other places in Kane's construction that "lose" a factor of $2^{O(d)}$. In all but one of these cases ${ }^{4}$, it's because Kane bootstraps the variance bound (1) using the hypercontractivity inequality in order to get control over $p$ 's behavior in various local neighborhoods. This hypercontractive inequality for degree- $d$ polynomials inherently loses $2 O(d)$ factors (see Theorem 3.10). By contrast, since our theorem already establishes the stronger hyperconcentration conclusion (2) (this is the second key improvement, bounding hypervariance rather than variance), we are able to provide argumentation that eliminates all of these $2^{O(d)}$ factors.

It should be noted that our Theorem 1.3 has one disadvantage compared to Kane's Theorem 1.2; the "with high probability over $\boldsymbol{x}$ " in our theorem is worse than in Kane's, and this leads to our seed length's worse dependence on $\varepsilon$.

We conclude this section by conjecturing that our Local Hyperconcentration Theorem remains true even at the larger scale of $\lambda=1 / \operatorname{poly}(d)$. If this were proven, it would immediately yield PRGs for Gaussian PTFs with seed length $\operatorname{poly}(d) \cdot \log n$. In fact, it seems possible to us that our Local Hyperconcentration Theorem holds not only in the context of degree- $d$ polynomials on Gaussian space, but even in the more general context of degree- $d$ polynomials on Boolean space $\{ \pm 1\}^{n}$. If this could be proven, it seems hopeful that our PRG would also extend to yield Boolean PRGs.

\subsection{Overview of the PRG Framework We Use}

We use the same PRG for Gaussian PTFs as in the prior works of Meka-Zuckerman PRG $[9,10]$ and Kane [3], namely

$$
z=\sqrt{\lambda} z_{1}+\sqrt{\lambda} z_{2}+\cdots+\sqrt{\lambda} z_{L},
$$

where the key parameter $\lambda$ is a small function of $d$ and $\varepsilon$, where $L=1 / \lambda$, and where $z_{1}, \ldots, z_{L}$ are independent random vectors, each having an $O(d)$-wise independent $n$-dimensional Gaussian

\footnotetext{
${ }^{4}$ Namely, our "noise insensitivity extension lemma" (Lemma 71 of the full version), where we eliminate a factor of $2^{O(d)}$ from the analogous result of Kane [4, Cor. 16].
} 
distribution. This leads to a seed length of essentially $O\left(d^{2} L \cdot \log n\right)$ (see Theorem 2.6), and hence all the effort goes into finding the largest $\lambda=\lambda(d, \varepsilon)$ such that Equation (3) $\varepsilon$-fools degree- $d$ Gaussian PTFs.

Here we review the Meka-Zuckerman and Kane works; our own analysis is heavily based on Kane's framework.

Meka-Zuckerman. The work of Meka and Zuckerman [9] gave PRGs for degree- $d$ Gaussian polynomial threshold functions with seed length $(d / \varepsilon)^{O(d)} \cdot \log n$. In fact, they also extended their results to Boolean PTFs, but we do not review that extension here. At a high level, their construction followed a basic two-part paradigm used both in the proof of Central Limit Theorems and in PRG construction: mollification + local low-degree behavior. To explain this, recall that we are trying to design a PRG $\mathcal{G}$ with

$$
\left|\mathbf{E}_{\boldsymbol{z} \sim \mathcal{G}}[f(\boldsymbol{z})]-\mathbf{E}_{\boldsymbol{x} \sim \mathcal{N}(0,1)^{n}}[f(\boldsymbol{x})]\right| \leq \varepsilon,
$$

where $f=\operatorname{sign}(p)$, with $p$ a degree- $d$ polynomial. Suppose first that we did not have the discontinuous "sign" function, but rather we just wanted the above inequality for $f=p$. In that case, it would suffice for the components of the random vector $z \sim \mathcal{G}$ to be " $d$-wise independent," and in fact this would achieve $\varepsilon=0$. Furthermore, there are standard techniques to produce an appropriate " $d$-wise independent" $G$ with seed length poly $(d) \cdot \log n$, which would be an excellent bound for us.

Of course, when we return to the actual scenario of $f=\operatorname{sign}(p)$, the function $f$ is not even a polynomial, let alone a low-degree one. The mollification portion of Meka and Zuckerman's work is to replace the sign function with a smooth approximator $\Phi$, which is equal to sign outside some interval $[-\lambda, \lambda]$. Because the sign function is scale-invariant $(\operatorname{sign}(t y)=\operatorname{sign}(y)$ for $t>0)$, we may normalize $p$ so that its variance $\operatorname{Var}[p(\boldsymbol{x})]$ is 1 . Then one chooses the parameter $\lambda=\Theta(\varepsilon / d)^{d}$. The smooth mollifier $\Phi$ will have derivatives of all orders, with $T$ th derivative $\Phi^{(T)}$ bounded in magnitude by $O\left(1 / \lambda^{T}\right)$. The replacement of sign by $\Phi$ leads to a mollication error of $O(\varepsilon)$, essentially due to the well-known anticoncentration bound for degree- $d$ Gaussian polynomials due to Carbery and Wright [1]: $\operatorname{Pr}\left[|p(\boldsymbol{x})| \leq(\varepsilon / d)^{d}\right] \leq O(\varepsilon)$. (Note also that thanks to a trick, this only needs to hold for $x$, and not the pseudorandom $z$.) With the mollifier in place, Meka and Zuckerman can try to bound

$$
\left|\mathbf{E}_{\boldsymbol{z} \sim \mathcal{G}}[\Phi(p(\boldsymbol{z}))]-\mathbf{E}_{\boldsymbol{x} \sim \mathcal{N}(0,1)^{n}}[\Phi(f(\boldsymbol{x}))]\right| \leq O(\varepsilon) .
$$

Now although $\Phi$ is not a polynomial, it is "locally a low-degree polynomial" (say, of degree 4), thanks to Taylor's theorem. The error in this statement scales like the 4 th derivative bound $\left\|\Phi^{(4)}\right\|_{\infty} \leq$ poly $(1 / \lambda)$, times the "locality scale". Thus as long as we substitute $O(1)$-wise independent Gaussians for true Gaussians at a "scale" of $\lambda^{\Theta(1)}$, we will not incur more than $O(\varepsilon)$ error. This sort of argumentation allows Meka and Zuckerman to show that the PRG in Equation (3) $\varepsilon$-fools degree- $d$ PTFs with $\lambda=\Theta(\varepsilon / d)^{d}$, which leads to their seed length of $(d / \varepsilon)^{O(d)} \cdot \log n$.

Kane. To repeat, our PRG analysis closely follows the structure of Kane's, which we now describe. Kane [4] shows that the PRG in Equation (3) succeeds with the improved (larger) value of $\lambda=$ $2^{-O(d)} \cdot \operatorname{poly}(\varepsilon)$, leading to his seed length of $2^{O(d)} \cdot \operatorname{poly}(1 / \varepsilon) \cdot \log n$. His "local concentration theorem" (Theorem 1.2) plays a central role in this, but he still needs to develop a complex framework (which we also employ) in order to complete the analysis.

Kane's Theorem 1.2 allows him to begin a new strategy for designing $\mathcal{G}$; rather than mollifying the sign function and taking $p(x)$ as a "black box" random variable, Kane instead mollifies the polynomial $p$ itself. Roughly speaking, Kane's strategy begins by replacing $p$ with $p \cdot \operatorname{Check}_{1}$, where $\operatorname{Check}_{1}(\widetilde{x})$ is a smoothed indicator function for the event that (1) holds at $\widetilde{x}$. The "with high probability over $\boldsymbol{x}$ " in Kane's Theorem 1.2 is in fact probability $1-\varepsilon$ provided $\lambda \leq 2^{-O(d)} \cdot \operatorname{poly}(\varepsilon)$, and this implies that the replacement of $p$ by $p \cdot$ Check $_{1}$ only incurs error $\varepsilon$. Now we may hope that the construction from Equation (3) will work; roughly, this requires that in a $\lambda$-scale neighborhood of every point $x$, say $\widetilde{\boldsymbol{x}}=\sqrt{1-\lambda} x+\sqrt{\lambda} \boldsymbol{w}$, the function $\operatorname{sign}(p) \cdot$ Check $_{1}$ is essentially determined by low-degree moments of $\boldsymbol{w}$. There are two cases. If $x$ is well into the region where $\operatorname{Check}_{1}(\widetilde{x})$ is 0 , then $p \cdot \operatorname{Check}_{1}$ is essentially 0 and $\operatorname{sign}(p) \cdot \operatorname{Check}_{1}$ is essentially constant. Otherwise, if $x$ is near the region where $\operatorname{Check}_{1}(\widetilde{x})$ is 1 , then by definition $\operatorname{Var}[p(\widetilde{x})]$ is very small. Thus $p$ is not varying very much in a neighborhood of $x$, and Taylor's theorem will tell us that low-degree moments suffice to essentially determine $p \cdot$ Check $_{1}$ in this neighborhood of $x$.

There are two catches here. First, the use of Taylor's theorem out to, say, degree 4 forces one to bound not just the expected squared deviation of $p$ from $|p(x)|$ in the $\lambda$-neighborhood of $\widetilde{x}$; it requires one to control, say, the 4 th-power deviation. This is where Kane uses the standard hypercontractivity-based fact that higherpower deviations can be controlled by the 2nd-power deviation (i.e., $\operatorname{Var}[p(\widetilde{\boldsymbol{x}})])$ at the expense of $2^{O(d)}$ losses. Kane is losing such factor anyway, since he takes $\lambda=2^{-O(d)} \cdot(1 / \varepsilon)$. (This is one place where our analysis takes advantage of the local hyperconcentration we prove in Theorem 1.3.)

The second catch is that Taylor's theorem needs to be applied not just to $p$ but to Check $_{1}$ itself. Now Check $_{1}$ is concerned with the variance of $p$ in a $\sqrt{\lambda}$-neighborhood of $x$. In order to control the Taylor error here, one needs to control the variance of the variance! Kane handles this by further mollifying $p$. He uses a generalization of Theorem 1.2 to show that at most points $x$, the variance of the variance in the neighborhood of $x$ is small. (We must prove a similar generalization of our Local Hyperconcentration Theorem; see Theorem 5.3.) Thus $p$ can be further mollified to $p \cdot \mathrm{Check}_{1} \cdot \mathrm{Check}_{2}$ at only small loss. Now we have three cases to consider when analyzing $p \cdot$ Check $_{1} \cdot$ Check $_{2}$; if $x$ is well into the region where Check is $_{2}$, then the mollified function is essentially 0 on the $\lambda$-neighborhood. Else, the variance of the variance of $p$ in the neighborhood is suitably small. Next, if $x$ is well into the region where Check $_{1}$ is 0 , then the mollified function is again essentially 0 on the neighborhood; otherwise, the variance of $p$ in the neighborhood is suitably small. In this third case, we are again in good shape to apply Taylor to $p$, and $\mathrm{Check}_{1} \ldots$ but to handle Taylor error for $\mathrm{Check}_{2}$, we need to introduce another check that the variance of the variance of the variance is small. Indeed, Kane's final mollifier needs not only this "descending" sequence of checks (that we will picture "vertically"), but for technical reasons needs additional "horizontally proliferating" checks (which, to avoid further lengthening this description, we will not discuss here). 
Luckily, all of these proliferating checks eventually "bottom out". The vertically descending checks bottom out because the " $i$-fold variance" is a polynomial of degree $d-i$, and hence the $(d+1)$-fold variance is constantly 0 . The horizontally proliferating checks may eventually be terminated due to the fact that a degree- $d$ polynomial is determined by its values at $d+1$ points. (Actually, one needs a quantitative version of this fact. Kane provides one involving another factor of $2^{O(d)}$; we eliminate this factor in our "noise insensitivity extension lemma," Lemma 71 of the full version.)

Kane's mollifier multiplies $p$ by poly $(d)$ many "Check $i, j$ " functions: one needs a generalization of Theorem 1.2 and another theorem to show that the mollification is close to $p$ at almost all points; and, when using Taylor's theorem at $x$, one needs a poly $(d)$-case analysis looking at the "deepest" check (if any) that "fails." If any check "fails," then the mollified function is essentially 0 ; otherwise, if they all pass, then in the $\lambda$-neighborhood of $z$, the variance of $p$, and the variance of the variance, and the variance of the variance of the variance, etc., are all suitably small for use in Taylor's theorem.

\section{THE HIGH-LEVEL STRUCTURE OF OUR PROOF}

Throughout this paper $p: \mathbb{R}^{n} \rightarrow \mathbb{R}$ is a nonzero polynomial of degree at most $d$, and we are interested in the degree- $d$ polynomial threshold function $f(x)=\operatorname{sign}(p(x))$. For a given $0<\varepsilon$ PRG $<1$, we determine a small value

$$
\underline{\lambda}:=\left(\frac{\varepsilon \mathrm{PRG}}{d}\right)^{O(\log d)}
$$

and we also let

$$
L:=1 / \underline{\lambda}, \quad k_{\text {indep }}:=\Theta(d) .
$$

Our main goal is:

Theorem 2.1 (MAIn Result: SUM of $k_{\text {indep-Wise IndePENDENT }}$ GAUSSIANS FOOLS DEGREE- $d$ PTFs). Let $\boldsymbol{x} \sim \mathcal{N}(0,1)^{n}$ be a standard $n$-dimensional Gaussian random vector, and let $z_{1}, \ldots, z_{L}$ be independent $k_{\mathrm{indep}}$-wise independent Gaussian random vectors in $\mathbb{R}^{n}$. Write

$$
Z:=\sqrt{\underline{\lambda}} z_{1}+\sqrt{\underline{\lambda}} z_{2}+\cdots+\sqrt{\underline{\lambda}} z_{L} .
$$

Then $|\mathrm{E}[\operatorname{sign}(f(Z))]-\mathbf{E}[\operatorname{sign}(f(\boldsymbol{x}))]| \leq \varepsilon_{\mathrm{PRG}}$.

To prove Theorem 2.1, we will construct a certain function

$$
\text { Mollifier }_{p}: \mathbb{R}^{n} \rightarrow[0,1],
$$

which is a smoothed indicator function for a collection of events (related to local hyperconcentration of $p$ ) that are expected to almost always occur. We then show the following:

THEOREM 2.2 (MOLLIFICATION ERROR THEOREM, ANALOGUE OF LEMMA 17 OF [4]).

$$
\operatorname{Pr}_{\boldsymbol{x} \sim \mathcal{N}(0,1)^{n}}\left[\operatorname{Mollifier}_{p}(\boldsymbol{x}) \neq 1\right] \leq \varepsilon_{\mathrm{PRG}} / 4 .
$$

We then extend the mollifier to take into account the sign of $p$ :

Definition 2.3. Define $I_{+}: \mathbb{R}^{n} \rightarrow[0,1]$ by

$$
I_{+}(x)=\operatorname{Mollifier}_{p}(x) \cdot \mathbf{1}[\operatorname{sign}(p(x))=1],
$$

and define $I_{-}$similarly as $I_{-}(x)=\operatorname{Mollifier}_{p}(x) \cdot 1[\operatorname{sign}(p(x))=-1]$.
The main thing we prove about $I_{ \pm}$is the following:

Theorem 2.4 (One Step of the Replacement Method, ANALOGUE of LemMA 19 of [4]). Fix any $x \in \mathbb{R}^{n}$, and assume the $\mathbb{R}^{n}$-valued random vectors $z, z^{\prime}$ are each $k_{\text {indep }}$-wise independent $n$-dimensional Gaussian vectors. Then we have

$$
\left|\mathrm{E}\left[I_{+}(\sqrt{1-\underline{\lambda}} x+\sqrt{\underline{\lambda}} z)\right]-\mathrm{E}\left[I_{+}\left(\sqrt{1-\underline{\lambda}} x+\sqrt{\underline{\lambda}} z^{\prime}\right)\right]\right| \leq \frac{\varepsilon_{\mathrm{PRG}}}{4 L} .
$$

The analogous statement for I_ also holds.

From this, a "Replacement Method" argument easily yields the following:

Corollary 2.5. For $Z$ as in Theorem 2.1 and $\boldsymbol{x} \sim \mathcal{N}(0,1)^{n}$ we have

$$
\left.\mid \mathrm{E}\left[I_{+}(\boldsymbol{Z})\right)\right]-\mathrm{E}\left[I_{+}(\boldsymbol{x})\right] \mid \leq \varepsilon \mathrm{PRG} / 4
$$

and similarly for I-

Proof. We may view $\boldsymbol{x} \sim \mathcal{N}(0,1)^{n}$ as

$$
x=\sqrt{\underline{\lambda}} x_{1}+\sqrt{\lambda} x_{2}+\cdots+\sqrt{\lambda} x_{L},
$$

where $\boldsymbol{x}_{1}, \ldots, \boldsymbol{x}_{L} \sim \mathcal{N}(0,1)^{n}$ are independent. For $0 \leq t \leq L$, write

$$
\boldsymbol{w}_{t}=\sqrt{\underline{\lambda}} x_{1}+\cdots+\sqrt{\underline{\lambda}} x_{t}+\sqrt{\underline{\lambda}} z_{t+1}+\cdots+\sqrt{\underline{\lambda}} z_{L},
$$

so $\boldsymbol{w}_{0}=Z$ and $\boldsymbol{w}_{L}=\boldsymbol{x}$. Thus by telescoping,

$$
\left.\left.\left.\mid \mathrm{E}\left[I_{+}(Z)\right)\right]-\mathrm{E}\left[I_{+}(\boldsymbol{x})\right]\left|\leq \sum_{t=1}^{L}\right| \mathrm{E}\left[I_{+}\left(\boldsymbol{w}_{t-1}\right)\right)\right]-\mathbf{E}\left[I_{+}\left(\boldsymbol{w}_{t}\right)\right)\right] \mid .
$$

For a fixed $1 \leq t \leq L$, if we write

$$
v=\sqrt{\frac{\underline{\lambda}}{1-\underline{\lambda}}} x_{1}+\cdots+\sqrt{\frac{\lambda}{1-\underline{\lambda}}} x_{t-1}+\sqrt{\frac{\underline{\lambda}}{1-\underline{\lambda}}} z_{t+1}+\cdots+\sqrt{\frac{\underline{\lambda}}{1-\underline{\lambda}}} z_{L},
$$

then

$$
\begin{aligned}
& \left.\left.\mid \mathrm{E}\left[I_{+}\left(\boldsymbol{w}_{t-1}\right)\right)\right]-\mathrm{E}\left[I_{+}\left(\boldsymbol{w}_{t}\right)\right)\right] \mid \\
& =\left|\mathrm{E}\left[I_{+}\left(\sqrt{1-\underline{\lambda}} \cdot \boldsymbol{v}+\sqrt{\underline{\lambda}} z_{t}\right)\right]-\mathrm{E}\left[I_{+}\left(\sqrt{1-\underline{\lambda}} \cdot \boldsymbol{v}+\sqrt{\underline{\lambda}} x_{t}\right)\right]\right| .
\end{aligned}
$$

Since $z_{t}$ and $\boldsymbol{x}_{t}$ are each $k_{\text {indep-wise independent } n \text {-dimensional }}$ Gaussian vectors, Theorem 2.4 implies that $(7) \leq \varepsilon$ PRG $/(4 L)$. Putting this into (6) completes the proof.

With the above ingredients in place, Theorem 2.1 follows almost immediately:

Proof of Theorem 2.1. Since $\operatorname{sign}(p) \leq 1-2 I_{-}$pointwise,

$$
\begin{aligned}
& \mathrm{E}[\operatorname{sign}(p(Z))] \leq \mathrm{E}\left[1-2 I_{-}(Z)\right] \\
& \leq \mathrm{E}\left[1-2 I_{-}(\boldsymbol{x})\right]+\varepsilon_{\mathrm{PRG}} / 2 \leq \mathrm{E}[\operatorname{sign}(p(\boldsymbol{x}))]+\varepsilon_{\mathrm{PRG}},
\end{aligned}
$$

where the second inequality is thanks to Corollary 2.5 and the third is thanks to Theorem 2.2. The reverse direction, which lower bounds $\mathbf{E}[\operatorname{sign}(p(Z))]$ by $\mathbf{E}[\operatorname{sign}(p(\boldsymbol{x}))]-\varepsilon_{\mathrm{PRG}}$ using $I_{+}$, is similar.

Theorem 2.1 shows that a scaled sum of $k_{\text {indep }}$-wise independent Gaussians fools degree- $d$ PTFs, but such a random variable is not quite the desired PRG since perfectly generating even a single Gaussian random variable formally requires infinitely many random bits. However, the following construction of Kane tells us that for fooling degree- $d$ Gaussian PTFs, it essentially suffices to 
find the least $L$ such that they are fooled by sums of $L$ independent $k$-wise Gaussians; then, one gets an explicit PRG with seed length $O(k L \cdot d \log n)$.

Theorem 2.6 (Section 6 of [4]). Let $n, d \in \mathbb{N}, 0<\varepsilon<1$. Suppose that for some $k, L \in \mathbb{N}$, degree-d Gaussian PTFs are $(\varepsilon / 2)$ fooled by $\sqrt{\lambda} z_{1}+\cdots+\sqrt{\lambda} z_{L}$, where $\lambda=1 / L$ and $z_{1}, \ldots, z_{L}$ are $k$-wise independent $n$-dimensional Gaussians. Then there is an explicit PRG for $\varepsilon$-fooling degree-d n-dimensional Gaussian PTFs with seed length

$$
O(k L \cdot d \log (d L n / \varepsilon)),
$$

which is simply $O(k L \cdot d \log n)$ under the reasonable assumptions that $d, 1 / \varepsilon, L \leq \operatorname{poly}(n)$.

As Kane does not quite explicitly state Theorem 2.6, we outline a proof in the appendix of the full version of this paper for completeness. Theorem 1.1 follows immediately from Theorem 2.1 and Theorem 2.6.

The remaining tasks are to define Mollifier $p$ and prove Theorems 2.2 and 2.4. We define Mollifier $p$ in Section 4 and prove Theorem 2.2 in Section 4.4. The proof of Theorem 2.4 is deferred to the full version of this paper.

\section{PROBABILISTIC PRELIMINARIES}

In this section we introduce notation and collect several probabilistic facts we will use. Throughout, boldface is used to indicate random variables, $\mathcal{N}(0,1)$ denotes the standard Gaussian (normal) distribution, and $\mathcal{N}(0,1)^{n}$ is the associated $n$-dimensional product distribution.

\subsection{Bits, Gaussians, and $k$-wise Independence}

Although this work is mainly concerned with Gaussian random variables, many (but not all) of the tools in it "generalize" to Boolean \pm 1 random variables. In order to illustrate this, we will provide some definitions and notations in this section that work in both cases. However the Boolean results are never strictly needed in this work, and the reader may prefer to ignore them and focus only on the Gaussian case.

The fact that PTFs over Boolean space generalize PTFs over Gaussian space holds because, for large $M$ and $\boldsymbol{x}^{(1)}, \ldots, \boldsymbol{x}^{(n)} \sim$ $\{ \pm 1\}^{M}$ uniform and independent,

$$
\boldsymbol{y}=\left(\frac{1}{\sqrt{M}} \sum_{i=1}^{M} \boldsymbol{x}_{i}^{(1)}, \ldots, \frac{1}{\sqrt{M}} \sum_{i=1}^{M} \boldsymbol{x}_{i}^{(n)}\right)
$$

is "close" to having an $\mathcal{N}(0,1)^{n}$ distribution, and because a degree- $d$ polynomial $p(\boldsymbol{y})$ is also a degree- $d$ polynomial in the $\boldsymbol{x}_{j}^{(i)}$, s. One sense of "closeness" here is that each $\boldsymbol{y}_{i}$ may be coupled with a true Gaussian $z_{i} \sim \mathcal{N}(0,1)$ in such a way that $\left|\boldsymbol{y}_{i}-z_{i}\right| \leq \frac{1}{\sqrt{M}}$ except with probability at most $O\left(\frac{1}{\sqrt{M}}\right)$.

Definition 3.1. Let $\mathcal{D}$ be a probability distribution on $\mathbb{R}$. We say that a random vector $z$ on $\mathbb{R}^{n}$ has a $k$-wise independent $\mathcal{D}$ distribution if each $z_{i}$ has distribution $\mathcal{D}$, and for all choices of $k$ indices $1 \leq i_{1}, \ldots, i_{k} \leq n$, the random variables $z_{i_{1}}, \ldots, z_{i_{k}}$ are independent. Examples include $\mathcal{D}$ being the uniform distribution on $\{ \pm 1\}$ (" $k$-wise independent bits") and the main concern in this paper, $\mathcal{D}$ being $\mathcal{N}(0,1)$ (" $k$-wise independent Gaussians").
Remark 3.2. The main way we use $k$-wise independence is to say that if $\boldsymbol{x}$ is $n$-wise independent, $z$ is $k$-wise independent, and $p: \mathbb{R}^{n} \rightarrow \mathbb{R}$ is a polynomial of degree at most $k$, then $\mathrm{E}[p(z)]=$ $\mathrm{E}[p(\boldsymbol{x})]$.

\subsection{Polynomial Expansions}

We recall standard facts and notation from analysis of Boolean functions and Hermite polynomials; see, e.g., [11] for a reference, and in particular [11, Ch. 11.2] for Hermite analysis.

Every function $g:\{ \pm 1\}^{n} \rightarrow \mathbb{R}$ can be represented by a multilinear polynomial,

$$
g(x)=\sum_{\alpha \in\{0,1\}^{n}} \widehat{g}(\alpha) x^{\alpha},
$$

where each $\widehat{g}(\alpha) \in \mathbb{R}$ and we use the standard multi-index notation $x^{\alpha}=\prod_{i=1}^{n} x_{i}^{\alpha_{i}}$ and $|\alpha|=\sum_{i} \alpha_{i}$. In "Gaussian space" the only functions we will ever analyze are polynomials; every degree- $d$ polynomial $g: \mathbb{R}^{n} \rightarrow \mathbb{R}$ can be written in Hermite polynomial decomposition as

$$
g(x)=\sum_{\substack{\alpha \in \mathbb{N}^{n} \\|\alpha| \leq d}} \widehat{g}(\alpha) h_{\alpha}(x)
$$

where each $\widehat{g}(\alpha) \in \mathbb{R}$, and the multivariate Hermite polynomial polynomial $h_{\alpha}$ is given by $h_{\alpha}(x)=h_{\alpha_{1}}\left(x_{1}\right) \cdots h_{\alpha_{n}}\left(x_{n}\right)$, where $h_{k}=\frac{1}{\sqrt{k !}} H_{k}$ is a normalized version of the univariate degree- $k$ "probabilists' Hermite polynomial" $H_{k}$. The multivariate Hermite polynomials $h_{\alpha}$ are orthonormal under $\mathcal{N}(0,1)^{n}$. Also, in the notation of Equation (8),

$$
\sum_{\substack{\alpha \in\{0,1\}^{n} \\|\alpha|=k}}\left(\frac{1}{\sqrt{M}} x^{(i)}\right)^{\alpha} \stackrel{M \rightarrow \infty}{\longrightarrow} h_{k}\left(z_{i}\right), \quad z \sim \mathcal{N}(0,1) .
$$

Let $g$ denote either an $n$-variate Boolean or Gaussian polynomial. We use standard notation $\mathrm{E}[g]$ for its mean (that is, $\mathrm{E}[g(\boldsymbol{x})]$ for $\boldsymbol{x} \sim\{ \pm 1\}^{n}$ in the former case, $\boldsymbol{x} \sim \mathcal{N}(0,1)^{n}$ in the latter), $\|g\|_{r}=$ $\mathbf{E}\left[|g(\boldsymbol{x})|^{r}\right]^{1 / r}$ for its $r$-norm $(r \geq 1)$, and $\operatorname{Var}[g]=\mathbf{E}\left[g^{2}\right]-\mathbf{E}[g]^{2}$ for its variance. It holds that

$$
\begin{gathered}
\mathbf{E}[g]=\widehat{g}(0), \quad \mathbf{E}\left[g^{2}\right]=\sum_{\alpha} \widehat{g}(\alpha)^{2}, \\
\text { hence } \operatorname{Var}[g]=\sum_{\alpha \neq 0^{n}} \widehat{g}(\alpha)^{2} .
\end{gathered}
$$

We write $g^{<k}=\sum_{|\alpha|<k} \widehat{g}(\alpha) h_{\alpha}$ for $k \in \mathbb{N}$, and similarly write $g^{=k}$ and $g^{\geq k}$. We also write $\mathrm{W}^{<k}[g]=\mathbf{E}\left[\left(g^{<k}\right)^{2}\right]=\sum_{|\alpha|<k} \widehat{g}(\alpha)^{2}$ for the "weight of $g$ below level $k$ ", and similarly write $\mathrm{W}^{=k}[g]$ and $\mathrm{W}^{\geq k}[g]$.

\subsection{Noise and Zooms}

A basic fact about Gaussians is that if $\boldsymbol{x}, \boldsymbol{y} \sim \mathcal{N}(0,1)^{n}$ are independent and $0 \leq \lambda \leq 1$, then $\sqrt{1-\lambda} \boldsymbol{x}+\sqrt{\lambda} \boldsymbol{y}$ is also distributed as $\mathcal{N}(0,1)$. In this work, $\lambda$ typically denotes a "small" quantity; for fixed $x \in \mathbb{R}^{n}$ we view $\sqrt{1-\lambda} x+\sqrt{\lambda} y$ as a " $\lambda$-noisy" version of $x$, and we view changing a polynomial $g$ 's input from $x \sim \mathcal{N}(0,1)^{n}$ to $\sqrt{1-\lambda} x+\sqrt{\lambda} \boldsymbol{y}$ as "zooming into $g$ at $x$ with scale $\lambda$ ". We make a precise definition: 
Definition 3.3. For $g$ an $n$-variate Gaussian polynomial, $0 \leq \lambda \leq 1$, and $x \in \mathbb{R}^{n}$, we define the function $g_{\lambda \mid x}$ by

$$
g_{\lambda \mid x}(y)=g(\sqrt{1-\lambda} x+\sqrt{\lambda} y)
$$

The function $g_{\lambda \mid x}(y)$ is a polynomial in $y$ of the same degree as $g$, and we (nonstandardly) refer to it as the $\lambda$-zoom of $g$ at $x$.

Remark 3.4. Referring again to Equation (8), one may verify that a $\lambda$-zoom of $g$ at a random $x$ is the Gaussian analogue of a standard Boolean concept: a random restriction of a function $g:\{ \pm 1\}^{n} \rightarrow$ $\mathbb{R}$ at $x \in\{ \pm 1\}^{n}$, meaning a subfunction obtained by proceeding through each coordinate $i$, and either fixing the $i$ th input to be $x_{i}$ with probability $1-\lambda$, or else leaving it unfixed ("free") with probability $\lambda$.

The fact that random restrictions of a Boolean function interact well with its polynomial expansion is well known; e.g. [11, Prop. 4.17] gives a formula for the expected square of any Fourier coefficient of a Boolean function under a random restriction. Carefully taking the "Gaussian special case" of this (using Equation (9)) yields the below analogue for random zooms. For completeness, we give a self-contained proof of this analogue in the appendix of the full version of this paper.

Proposition 3.5. For $g: \mathbb{R}^{n} \rightarrow \mathbb{R}$ a polynomial, $0 \leq \lambda \leq 1$, and $\beta \in \mathbb{N}^{n}$,

$$
\underset{\boldsymbol{x} \sim \mathcal{N}(0,1)^{n}}{\mathbf{E}}\left[\widehat{g \lambda \mid x}(\beta)^{2}\right]=\sum_{\gamma \geq \beta} \operatorname{Pr}[\operatorname{Bin}(\gamma, \lambda)=\beta] \widehat{g}(\gamma)^{2},
$$

where $\operatorname{Bin}(\gamma, \lambda)$ denotes an $n$-dimensional random vector with independent components, the jth of which is distributed as the binomial random variable $\operatorname{Bin}\left(\gamma_{j}, \lambda\right)$.

Summing the above proposition over all multi-indices $\beta$ of a given weight $|\beta|=m$ immediately yields the following useful corollary:

Corollary 3.6. For $g: \mathbb{R}^{n} \rightarrow \mathbb{R}$ a polynomial, $0 \leq \lambda \leq 1$, and $m \in \mathbb{N}$,

$$
\underset{\boldsymbol{x} \sim \mathcal{N}(0,1)^{n}}{\mathrm{E}}\left[\mathrm{W}^{=m}\left[g_{\lambda \mid \boldsymbol{x}}\right]\right]=\sum_{M} \operatorname{Pr}[\operatorname{Bin}(M, \lambda)=m] \cdot \mathrm{W}^{=M}[g] .
$$

\subsection{Noise Operator and Hypercontractivity}

Considering the mean of the zoom of a polynomial leads to the "Gaussian noise" (or "Ornstein-Uhlenbeck") operator (see, e.g., [11, Def. 11.12]):

Definition 3.7. Given $0<\rho \leq 1$, the operator $\mathrm{U}_{\rho}$ acts on Gaussian polynomials $g: \mathbb{R}^{n} \rightarrow \mathbb{R}$ via

$$
\begin{aligned}
\left(\mathrm{U}_{\rho} g\right)(x) & \left.=\underset{\boldsymbol{y} \sim \mathcal{N}(0,1)^{n}}{\mathbf{E}}\left[g\left(\rho x+\sqrt{1-\rho^{2}} \boldsymbol{y}\right)\right)\right] \\
& =\underset{\boldsymbol{y} \sim \mathcal{N}(0,1)^{n}}{\mathbf{E}}\left[g_{\left(1-\rho^{2}\right) \mid x}(\boldsymbol{y})\right] .
\end{aligned}
$$

It is well known that $\mathrm{U}_{\rho}$ acts diagonally in the Hermite polynomial basis $\left(h_{\alpha}\right)_{\alpha \in \mathbb{N}^{n}}$ :

$$
\mathrm{U}_{\rho} g=\sum_{\alpha \in \mathbb{N}^{N}} \rho^{|\alpha|} \widehat{g}(\alpha) h_{\alpha} .
$$

In particular, if $g$ is a degree- $d$ polynomial, so too is $\mathrm{U}_{\rho} g$.
We may also write $\mathrm{U}_{\rho}$ for the analogous Boolean noise operator (more usually denoted $\mathrm{T}_{\rho}$, see [11, Def. 2.46]), definable for $g:\{ \pm 1\}^{n} \rightarrow \mathbb{R}$ either through Equation (10), or by stipulating that $\mathrm{U}_{\rho} g(x)$ is the mean of a random restriction of $g$ at $x$ with $\rho$-probability of fixing a coordinate.

Finally, somewhat unusually, we will need to extend the definition of $\mathrm{U}_{\rho}$ to $\rho>1$, which we can do via the formula Equation (10); equivalently, by stipulating that $\mathrm{U}_{\rho^{-1}}=\mathrm{U}_{\rho}^{-1}$. For $\rho>1$ this operator no longer has a "probabilistic interpretation", but it still maps degree- $d$ polynomials to degree- $d$ polynomials.

Remark 3.8. We will several times use the "semi-group property", $\mathrm{U}_{\rho_{1}} \mathrm{U}_{\rho_{2}}=\mathrm{U}_{\rho_{1} \rho_{2}}$, which is immediate from Equation (10).

At one point in our analysis we will also need the notion of Gaussian noise stability:

Definition 3.9. For $g: \mathbb{R}^{n} \rightarrow \mathbb{R}$ and $\rho>0$,

$$
\operatorname{Stab}_{\rho}[g]=\underset{\boldsymbol{y} \sim \mathcal{N}(0,1)^{n}}{\mathbf{E}}\left[g(\boldsymbol{y}) \cdot \mathrm{U}_{\rho} g(\boldsymbol{y})\right]=\sum_{\alpha} \rho^{|\alpha|} \widehat{g}(\alpha)^{2},
$$

where the last equality is by Equation (10) and orthonormality of Hermite polynomials.

Hypercontractivity. A nontrivial and highly useful property of the Boolean/Gaussian noise operator $\mathrm{U}_{\rho}$ is hypercontractivity (see, e.g., $[11$, Secs. 9.2, 11.1]):

THEOREM $3.10((2, q)$-HyPerContractive InEQUALITy). Let $g$ be a Gaussian or Boolean polynomial. Then $\left\|\mathrm{U}_{1 / \sqrt{q-1}} g\right\|_{q} \leq\|g\|_{2}$ holds for any $q>2$.

Hypercontractivity has the following consequences (see [11, Thms. 9.22, 9.23]):

TheOREM 3.11. Let $g$ be a Gaussian or Boolean polynomial of degree at most $k$. Then $\|g\|_{2} \leq e^{k}\|g\|_{1}$.

TheOREM 3.12. Let $g$ be a Gaussian or Boolean polynomial of degree at most $k$. Then for any $t \geq \sqrt{2 e}^{k}$,

$$
\underset{\boldsymbol{x}}{\operatorname{Pr}}\left[|g(\boldsymbol{x})| \geq t\|g\|_{2}\right] \leq \exp \left(-\frac{k}{2 e} t^{2 / k}\right) .
$$

\subsection{Hyperconcentration: Our Key Tool}

The ideas in this section, though technically standard, are part of the conceptual contribution of this work.

Very often we will need to show that a random variable is tightly concentrated around its mean in a multiplicative sense. Let us start with some notation.

Notation 3.13. We use the following notation to denote that two reals $a, b>0$ are multiplicatively close: For $v \geq 0$,

$$
a \approx_{v} b \quad e^{-v} \leq a / b \leq e^{v} .
$$

Note that this condition is indeed symmetric in $a$ and $b$. We extend the notation to all $a, b \in \mathbb{R}$ by stipulating that $a \approx_{v} b$ if: $a b>0$ and the above condition holds; or, $a=b=0$.

Given a real random variable $w$ with mean $\mu$, a standard way to show that $\boldsymbol{w} \approx \mu$ with high probability is to first establish $\operatorname{stddev}[w] \leq \eta|\mu|$ and then use Chebyshev's inequality. When this holds we informally say that $\boldsymbol{w}$ concentrates around its mean. In 
this work, a crucial concept will be improving this concentration using higher norms.

Definition 3.14. Let $q>2$ and $\eta \geq 0$ be real numbers. We say a real random variable $\boldsymbol{w}$ with mean $\mu$ is $(q, \eta)$-hyperconcentrated if

$$
\|\boldsymbol{w}-\mu\|_{q}=\mathrm{E}\left[|\boldsymbol{w}-\mu|^{q}\right]^{1 / q} \leq \eta|\mu| .
$$

The utility of this definition is that it gives an improvement to the Chebyshev inequality:

Proposition 3.15. Suppose that random variable $w$ with mean $\mu$ is $(q, \eta)$-hyperconcentrated. Then for any $t>0$, except with probability at most $(\eta / t)^{q}$ we have $|w-\mu| \leq t|\mu|$ (and in particular $w \approx_{2 t} \mu$ ift $\leq 1 / 2)$. $\left.\mu\right|^{q}$.

Proof. Apply Markov's inequality to the random variable $\mid \boldsymbol{w}-$

We'll also need the following simple consequence of hyperconcentration:

LEMMA 3.16. Suppose $z$ is an $\mathbb{R}^{m}$-valued random vector with $(q, \eta)$-hyperconcentrated components, and write $\mu=\mathrm{E}[z]$. Then for any multi-index $\alpha \in \mathbb{N}^{m}$ with $|\alpha| \leq q$,

$$
\mathrm{E}\left[|z-\mu|^{\alpha}\right] \leq \eta^{|\alpha|}|\mu|^{\alpha} .
$$

Proof. We have

$$
\begin{aligned}
\mathrm{E}\left[|z-\mu|^{\alpha}\right] & =\mathrm{E}\left[\prod_{i=1}^{n}\left|z_{i}-\mu_{i}\right|^{\alpha_{i}}\right] \leq \prod_{i=1}^{n} \mathrm{E}\left[\left|z_{i}-\mu_{i}\right|^{\alpha_{i} \cdot \frac{|\alpha|}{\alpha_{i}}}\right]^{\frac{\alpha_{i}}{|\alpha|}} \\
& \leq \prod_{i=1}^{n}\left(\eta \mu_{i}\right)^{\alpha_{i}}=\eta^{|\alpha|}|\mu|^{\alpha},
\end{aligned}
$$

where the first inequality is from Hölder's inequality and the second is from Definition 3.14.

The random variables we'll show hyperconcentration for will be Gaussian polynomials. We will do this by bounding a quantity that we term their "hypervariance", and that plays a central role in our work:

Definition 3.17. Let $g$ be a Gaussian or Boolean polynomial. Then for $R>1$, we define the $R$-hypervariance of $g$ to be

$$
\operatorname{HyperVar}_{R}[g]:=\operatorname{Var}\left[\mathrm{U}_{R} g\right]=\sum_{\alpha \neq 0} R^{2|\alpha|} \widehat{g}(\alpha)^{2} .
$$

(For $R=1$, this reduces to the usual variance of $g$.)

LEMmA 3.18. Let $g$ be a Gaussian or Boolean polynomial. Write $\mu=$ $\mathrm{E}[g]$ and assume $\operatorname{HyperVar}_{R}[g] \leq \theta \mu^{2}$. Then the random variable $g(\boldsymbol{x})$ is $\left(1+R^{2}, \sqrt{\theta}\right)$-hyperconcentrated.

Proof. Writing $\bar{g}=g-\mu$, our hypothesis is that $\left\|\mathrm{U}_{R} \bar{g}\right\|_{2}^{2} \leq \theta \mu^{2}$. By hypercontractivity, we have

$$
\|\bar{g}\|_{1+R^{2}}=\left\|\mathrm{U}_{1 / R} \mathrm{U}_{R} \bar{g}\right\|_{1+R^{2}} \leq\left\|\mathrm{U}_{R} \bar{g}\right\|_{2} .
$$

Thus $\|\bar{g}\|_{1+R^{2}} \leq \sqrt{\theta}|\mu|$, as needed.
The hypothesis in Lemma 3.18, that $g$ 's hypervariance is small compared to its squared-mean, will be an important one for us. It is essentially the same as the hypothesis that $g$ 's hypervariance is small compared to its squared-2-norm (since squared-2-norm equals squared-mean plus variance, and hypervariance is at least variance for all $R>1$ ). It will be slightly more convenient in our Local Hypervariance Theorem to work with the latter hypothesis, so we codify it here and establish the analogue of Lemma 3.18.

Definition 3.19. Let $g$ be a Gaussian or Boolean polynomial, and let $R>1$. We say that $g$ is $(R, \varepsilon)$-attenuated if $\operatorname{HyperVar}_{R}[g] \leq$ $\varepsilon\|g\|_{2}^{2}$.

Remark 3.20. Intuitively, a polynomial $g$ is attenuated if for each $i \geq 1$, the amount of Hermite weight it has at level $i$ is "very small" compared with the total Hermite weight (squared 2-norm) of $g$. Crucially, the precise quantitative definition of "very small" in the preceding sentence depends on the weight level $i$, and gets exponentially stronger (smaller) as $i$ gets larger. An intuition which may possibly be helpful is to think of an attenuated polynomial as a polynomial which is "morally constant" over Gaussian space.

Returning to hyperconcentration, we have the following:

LEMMA 3.21. Let $g$ be a Gaussian or Boolean polynomial that is $(R, \theta)$-attenuated, with $R \geq \sqrt{2}$ and $\theta \leq 1$. Then the random variable $g(\boldsymbol{x})$ is $\left(1+\frac{1}{2} R^{2}, \sqrt{\theta}\right)$-hyperconcentrated.

Proof. Using the notation $\mu$ and $\bar{g}$ again, and starting with the $(R, \theta)$-attenuation assumption, we have

$$
\begin{gathered}
\sum_{j \geq 1} R^{2 j}\left\|g^{=j}\right\|_{2}^{2} \leq \theta\left(\mu^{2}+\sum_{j \geq 1}\left\|g^{=j}\right\|_{2}^{2}\right) \\
\Longrightarrow \sum_{j \geq 1}\left(R^{2 j}-\theta\right)\left\|g^{=j}\right\|_{2}^{2} \leq \theta \mu^{2} \\
\Longrightarrow \sum_{j \geq 1}\left(\frac{R}{\sqrt{2}}\right)^{2 j}\left\|g^{=j}\right\|_{2}^{2} \leq \theta \mu^{2},
\end{gathered}
$$

where the last step used $R \geq \sqrt{2}$ and $\theta \leq 1$. But this is equivalent to HyperVar $_{\frac{R}{\sqrt{2}}}[g(\boldsymbol{x})] \leq \theta \mu^{2}$, so the result follows from Lemma 3.18.

Combining this with Lemma 3.21 and Proposition 3.15 yields the following useful result, which informally says that "attenuated polynomials are very likely to take values multiplicatively close to their means":

Proposition 3.22. Let $g$ be a Gaussian or Boolean polynomial that is $(R, \theta)$-attenuated, with $R \geq \sqrt{2}$ and $\theta \leq 1$. Write $\mu=\mathrm{E}[g]$. Then assuming $0<\gamma \leq 1$, we have $g(\boldsymbol{x}) \approx \gamma \mu$ except with probability at most $(2 \sqrt{\theta} / \gamma)^{\frac{1}{2} R^{2}+1}$.

\subsection{Special Properties of Gaussian Random Variables}

All of the results in this section so far have applied equally well to Gaussian or Boolean polynomials. We now give the two results we will use that are specific just to Gaussian polynomials. The first is a well known result of Carbery and Wright [1] on anticoncentration (see e.g. [4, Lem. 23], [11, Sec. 11.6]): 
TheOrEm 3.23 (GAussian CARBERY-Wright). There is a univeral constant $C$ such that for any degree-d polynomial $g: \mathbb{R}^{n} \rightarrow \mathbb{R}$ and any $0 \leq \delta \leq 1$,

$$
\operatorname{Pr}_{\boldsymbol{x} \sim \mathcal{N}(0,1)^{n}}\left[|g(\boldsymbol{x})|<\left(\frac{\delta}{C \cdot d}\right)^{d} \cdot\|g\|_{2}\right] \leq \delta .
$$

The second Gaussian-specific result we use is a key lemma from Kane's work [4, Lemma 9]. This lemma was the essential ingredient he used to prove his "local concentration" result Theorem 1.2. At first glance, it may look much stronger than Theorem 1.2, because it gives a nontrivial kind of concentration result even for $\lambda$ as large as $1 / \operatorname{poly}(d)$. However the concentration one gets in (almost all) local neighborhoods is somewhat weak: one gets that $g_{\lambda \mid x}$ 's values are with high probability near a specific value, but this is not enough to even conclude that $\operatorname{std\operatorname {dev}}\left[g_{\lambda \mid x}\right]$ is small compared to that value. Kane uses hypercontractivity to bootstrap this to control over the variance when he obtains Theorem 1.2, and this loses a $2^{O(d)}$ factor. When we employ Lemma 3.24 below, we will already be working with hyperconcentrated functions, which means we will not lose much when similarly bootstrapping.

Lemma 3.24. ([4, Lemma 9] with parameters renamed.) Let $g$ : $\mathbb{R}^{n} \rightarrow \mathbb{R}$ be a degree-d polynomial and let $0<\beta<1$. Then for $\boldsymbol{x}, \boldsymbol{y} \sim \mathcal{N}(0,1)^{n}$ independent, except with probability $\beta$ we have

$$
g_{\lambda \mid \boldsymbol{x}}(\boldsymbol{y}) \approx_{v} g(\boldsymbol{x}) \quad \text { for } v=O\left(d^{2} / \beta\right) \cdot \sqrt{\lambda}
$$

(provided $\lambda$ is small enough that $v \leq 1$ ).

Kane's proof of this lemma (seemingly) crucially relies on the rotational invariance of $n$-dimensional Gaussians.

\section{DEFINING Mollifier $r_{p}$}

The definition of Mollifier $p$ involves a collection $\subseteq$ of "statistics" of the polynomial $p$. Each statistic $s \in \mathcal{S}$ will be a certain nonnegative polynomial $s: \mathbb{R}^{n} \rightarrow \mathbb{R}^{\geq 0}$, defined in terms of $p$, of degree at most $2 d$.

The definition also involves a collection MollifierChecks of "mollifier checks". Each mollifier check in the set MOLLIFIERCHECKS will consist of two ingredients:

$$
\begin{gathered}
\text { Check }=(\operatorname{IneQ}, \delta), \\
\text { where IneQ }=\left(\gamma, s_{u}, s_{v}\right) \text { means " } s_{u} \geq \gamma s_{v} \text { " }
\end{gathered}
$$

for some statistics $s_{\mathcal{u}}, s_{\mathcal{v}} \in \mathbb{S}$ and some nonnegative value $\gamma$, and where $\delta \geq 0$ is a "softness" parameter. The intuitive meaning of СНEск applied at a point $x \in \mathbb{R}^{n}$ is that it is "softly" checking that $s_{u}(x) \geq \gamma s_{v}(x)$, up to a multiplicative factor of roughly $1 \pm \delta$. More precisely:

Definition 4.1. Let $\sigma: \mathbb{R} \rightarrow[0,1]$ be a smooth function satisfying

$$
\sigma(t)= \begin{cases}0 & \text { if } t \leq-1 \\ 1 & \text { if } t \geq+1\end{cases}
$$

and which is such that for all $j \leq d$, the magnitude of $\sigma$ 's $j$-th derivative is everywhere bounded by $j^{O(j)}$. (This is easily achieved by standard constructions such as taking $\sigma$ to be a suitable polynomial of degree $O(d)$ on the interval $[-1,1]$.) Also, given a mollifier check Check $\in$ MollifierChecks as in (11), define

$$
\text { SoftCheck }_{\text {CHEcK }}: \mathbb{R}^{n} \rightarrow[0,1]
$$

by

$$
\operatorname{SoftCheck}_{\text {CHECK }}(x)=\sigma\left(\delta^{-1} \ln \left(\frac{s_{u}(x)}{\gamma s_{v}(x)}\right)\right),
$$

where we take $0 / 0=+\infty$. We remark that

$$
\begin{aligned}
& \operatorname{SoftCheck}_{\text {Снеск }}(x)=1 \text { if } s_{u}(x) \geq \exp (\delta) \cdot \gamma s_{v}(x), \\
& \operatorname{SoftCheck}_{\text {CHеск }}(x)=0 \text { if } s_{u}(x) \leq \exp (-\delta) \cdot \gamma s_{v}(x) .
\end{aligned}
$$

The function Mollifier $p$ is the product of all the mollifier checks:

\section{Definition 4.2}

$$
\operatorname{Mollifier}_{p}(x)=\prod_{\text {CHECK } \in \text { MOLLIFIERCHeCKS }} \operatorname{SoftCheck}_{\text {Check }}(x) .
$$

To complete the definition of Mollifier $p$ we need to: (i) define the statistics in $\subseteq$; and, (ii) define the collection of mollifier checks, denoted MOLLIFIERCHEcKs. We do each of these in turn below.

\subsection{The Statistics in $\subseteq$}

4.1.1 Noisy Derivatives of Amplified Polynomials. Now we arrive at a novel definition in this work which plays a key role in our results. We will make extensive use of the following notion, which can be thought of as a sort of "noisy derivative of the $R$-amplified version of $g$ at $x$ in directions $y$ and $y^{\prime}$." This is a variant of one of the key definitions of [4] (the second definition in Section 3 of that paper) but with the crucial difference that now we consider the " $R$-amplified" version of $g$ in place of just $g$ itself as was the case in the corresponding definition in [4]:

Definition 4.3. Given two vectors $y, y^{\prime} \in \mathbb{R}^{n}$, define the operator $\Delta \stackrel{(R, \lambda)}{y, y^{\prime}}$ on polynomials $g: \mathbb{R}^{n} \rightarrow \mathbb{R}$ via

$$
\left(\Delta \frac{(R, \underline{\lambda})}{y, y^{\prime}} g\right)(x):=\frac{\mathrm{U}_{\underline{R}} g_{\underline{\lambda} \mid x}(y)-\mathrm{U}_{\underline{R}} g_{\underline{\lambda}} \mid x\left(y^{\prime}\right)}{\sqrt{2}} .
$$

We remark that $\mathrm{U}_{\underline{R}} g_{\underline{\lambda}} \mid x(y)$ is parenthesized as $\left(\mathrm{U}_{\underline{R}}\left(g_{\underline{\lambda}} \mid x\right)\right)(y)$.

The following is easily verified:

FACT 4.4. If $g: \mathbb{R}^{n} \rightarrow \mathbb{R}$ is a polynomial of degree at most $d$, then for every $y, y^{\prime} \in \mathbb{R}^{n}$, the function $\Delta \frac{(R, \lambda)}{y, y^{\prime}} g$ is a polynomial of degree at most $d-1$.

The following simple but crucial fact connects the derivative notion from Definition 4.3 to the hypervariance notion from Definition 3.17:

FACT 4.5. For fixed $x \in \mathbb{R}^{n}$ and independent $n$-dimensional Gaussians $\boldsymbol{y}, \boldsymbol{y}^{\prime} \sim \mathcal{N}(0,1)^{n}$, we have that

$$
\underset{\boldsymbol{y}, \boldsymbol{y}^{\prime}}{\mathbf{E}}\left[\left(\Delta \stackrel{(R, \underline{\lambda})}{\boldsymbol{y}, \boldsymbol{y}^{\prime}} g(x)\right)^{2}\right]=\operatorname{HyperVar}_{\underline{R}}\left[g_{\underline{\lambda}} \mid x\right] .
$$


Proof. The left-hand side is equal to

$$
\begin{aligned}
& \frac{1}{2} \mathbf{E}\left[\left(\sum_{\alpha} \underline{R}^{|\alpha|} \widehat{g_{\underline{\lambda} \mid x}}(\alpha)\left(h_{\alpha}(\boldsymbol{y})-h_{\alpha}\left(\boldsymbol{y}^{\prime}\right)\right)\right)^{2}\right] \\
& =\frac{1}{2} \mathbf{E}\left[\left(\sum_{\alpha \neq 0} \underline{R}^{|\alpha|} \widehat{g_{\underline{\lambda} \mid x}}(\alpha)\left(h_{\alpha}(\boldsymbol{y})-h_{\alpha}\left(\boldsymbol{y}^{\prime}\right)\right)\right)^{2}\right] \\
& =\frac{1}{2} \sum_{\alpha, \beta \neq 0} \underline{R}^{|\alpha|+|\beta|} \widehat{g_{\underline{\lambda} \mid x}}(\alpha) \widehat{g_{\underline{\lambda}} \mid x}(\beta) . \\
& =\sum_{|\alpha|>0} \underline{R}^{2|\alpha|} \widehat{E}_{\underline{\underline{\lambda} \mid x}}(\alpha)^{2}=\operatorname{HyperVar}_{\underline{R}}\left[\left(h_{\alpha}(\boldsymbol{y})-h_{\alpha}\left(\boldsymbol{y}^{\prime}\right)\right)\left(h_{\beta}(\boldsymbol{y})-h_{\beta}\left(\boldsymbol{y}^{\prime}\right)\right)\right]
\end{aligned}
$$

where the penultimate equality is by orthonormality of the Hermite polynomials and independence of $\boldsymbol{y}, \boldsymbol{y}^{\prime}$.

\subsubsection{The Statistics in $\subseteq$. Fix a parameter}

$$
\underline{R}:=\Theta(T)
$$

where $T$ is a parameter we will set later. We can now define the set of statistics, $\subseteq$. Each statistic is doubly indexed by a pair of natural numbers; there are $(d+1)(D+1)$ many statistics, $\left\{s_{i, j}\right\}_{0 \leq i \leq d, 0 \leq j \leq D}$, where

$$
D:=(2 d+1)^{2} .
$$

It is convenient for us to view the elements of $\subseteq$ as being arranged in a grid where the $(i, j)$-th statistic $s_{i, j}$ is in row $i$ and column $j$ (we will often use terminology of this sort). We remark that our statistic $s_{i, j}$ will closely correspond to the functions called $\left|p_{\theta}^{(\ell), m}(X)\right|_{2}^{2}$ in [4] $(i=\ell, j=m)$, except that as mentioned we use amplified noisy derivatives where Kane just had noisy derivatives.

All statistics are defined in terms of the underlying degree- $d$ polynomial $p$. We first define the $0^{\text {th }}$ column of statistics:

Definition $4.6\left(0^{t h}\right.$ column of statistics). For $i \in\{0, \ldots, d\}$, we define

$$
s_{i, 0}(x):=\mathrm{E}\left[\left(\Delta_{\boldsymbol{y}_{i}, \underline{\boldsymbol{y}_{i}^{\prime}}}^{(\underline{R}, \underline{\lambda})} \stackrel{(R, \underline{\lambda})}{\boldsymbol{y}_{i-1}, \boldsymbol{y}_{i-1}^{\prime}} \cdots \Delta_{\boldsymbol{y}_{1}, \underline{\boldsymbol{y}_{1}^{\prime}}}^{(R, \lambda)} p(x)\right)^{2}\right],
$$

where $\boldsymbol{y}_{1}, \boldsymbol{y}_{1}^{\prime}, \ldots, \boldsymbol{y}_{i}, \boldsymbol{y}_{i}^{\prime} \sim \mathcal{N}(0,1)^{n}$ are independent.

Remark 4.7. By Fact $4.4, s_{d, 0}$ is a constant function and $s_{d+1,0}$ is identically zero; this is why we consider $s_{i, 0}$ only for $i \leq d$.

For technical reasons, we will also need to use slight variants of the statistics $s_{i, 0}$, which correspond to taking an average over mildly noisy versions of the input:

Definition 4.8 (The remaining statistics). For $i \in\{0, \ldots, d\}$ and $j \in\{1, \ldots, D\}$ we define $s_{i, j}$ by

$$
s_{i, j}(x):=\mathrm{U}_{\sqrt{1-\underline{\lambda}}} s_{i, j-1}(x)=\mathbf{E}_{\boldsymbol{y} \sim \mathcal{N}(0,1)^{n}}\left[\left(s_{i, j-1}\right)_{\underline{\lambda} \mid x}(\boldsymbol{y})\right] .
$$

Remark 4.9. Using the semigroup property, we have that

$$
s_{i, j}(x)=\mathrm{U}_{(1-\underline{\lambda})^{j / 2}} s_{i, 0}(x)=\mathbf{E}_{\boldsymbol{y} \sim \mathcal{N}(0,1)^{n}}\left[\left(s_{i, 0}\right)_{1-(1-\underline{\lambda})^{j} \mid x}(\boldsymbol{y})\right] .
$$

This completes the formal definition of the statistics in $\subseteq$; however, it will be very useful for us to view the statistics from a different perspective based on distributions of polynomials. We introduce this perspective in the next subsection.

\subsection{A Distributional View on the Statistics}

For the sake of probabilistic technicalities, we will need to define a notion of a distribution of polynomials being "nice":

Definition 4.10. We say that a distribution $\left(g_{v}\right)_{v \sim \Upsilon}$ of $n$-variable polynomials of degree at most $d$ is nice if

- $\Upsilon$ is the normal distribution $\mathcal{N}(0,1)^{I}$ for some natural number $I$, and

- for each $v$ in the support of $\Upsilon$, the coefficients of $p_{v}$ are polynomials of degree at most $d$ in $v$.

It will be convenient for us to view the statistics $s_{i, j}$ as averages of squares of polynomials drawn from various nice distributions. To do this, we inductively define a grid of nice distributions of polynomials $\mathcal{F}_{i, j}$ as follows. The base distribution, $\mathcal{F}_{0,0}$ is just the probability distribution with a single outcome, namely the polynomial $p$. (Note that this corresponds to a nice distribution with $I=0$.) Next, we inductively define the distributions $\mathcal{F}_{i, 0}$ as follows:

- To make a draw from $\mathcal{F}_{i, 0}$, where $i>0$ : First draw $f \sim$ $\mathcal{F}_{i-1,0}$. Then draw $\boldsymbol{y}, \boldsymbol{y}^{\prime} \sim \mathcal{N}(0,1)^{n}$. Then output the polynomial $\Delta \underset{\boldsymbol{y}, \boldsymbol{y}^{\prime}}{(R,} \boldsymbol{f}$.

The following "operator notation" for zooms will be convenient: for a polynomial $p$ and a vector $y \in \mathbb{R}^{n}$, we define the notation

$$
\mathrm{N}_{y} p:=p_{1-\underline{\lambda} \mid y}
$$

With the distributions of polynomials $\mathcal{F}_{i, 0}$ defined as above, we inductively define the distributions $\mathcal{F}_{i, j}$, where $j>0$ as follows:

- To make a draw from $\mathcal{F}_{i, j}$, where $j>0$ : First draw $f \sim$ $\mathcal{F}_{i, j-1}$. Then draw $\boldsymbol{y} \sim \mathcal{N}(0,1)^{n}$. Then output the polynomial $\mathrm{N}_{\boldsymbol{y}} f$.

It is immediate from these definitions that each $\mathcal{F}_{i, j}$ is a nice distribution of polynomials of degree at most $d$. It is also immediate, comparing the above definition against Definition 4.6 and Definition 4.8, that for each $i \in\{0, \ldots, d\}$ and each $j \in\{0, \ldots, D\}$ we have that

$$
s_{i, j}=\mathrm{E}_{f \sim \mathcal{F}_{i, j}}\left[f^{2}\right]
$$

Finally, it is straightforward to check from these definitions (using also Fact 4.5) that

$$
\begin{aligned}
& s_{i+1,0}(x)=\mathrm{E}_{f \sim \mathcal{F}_{i, 0}}\left[\operatorname{HyperVar}_{\underline{R}}\left[f_{\underline{\lambda} \mid x}\right]\right], \\
& s_{i, j+1}(x)=\mathrm{E}_{f \sim \mathcal{F}_{i, j}}\left[\left\|f_{\underline{\lambda \mid x}}\right\|_{2}^{2}\right] .
\end{aligned}
$$

These characterizations will be useful when we analyze the statistics later.

\subsection{Defining the Mollifier Checks}

Intuition. In this subsection we define the collection of mollifier checks, denoted MolLIFIERCHEcks. Before formally defining these checks, we give some useful intuition concerning them. We will show in Section 4.4 that except with very small failure probability over $\boldsymbol{x} \sim \mathcal{N}(0,1)^{n}$, the statistics $s_{i, j}(\boldsymbol{x})$ satisfy the following properties, where $\hat{\lambda}, \delta_{\text {horz }}>0$ are suitable small parameters: 
(1) Local hyperconcentration: For each $i \in\{0, \ldots, d-1\}$,

$$
s_{i+1,0}(\boldsymbol{x}) \leq \widehat{\lambda} s_{i, 1}(\boldsymbol{x}) \text {. }
$$

(2) Insensitivity under noise: For each $i \in\{0, \ldots, d\}, j \in$ $\{0, \ldots, D-2\}$,

$$
s_{i, j}(\boldsymbol{x}) \approx_{\delta_{\mathrm{horz}}} s_{i, j+1}(\boldsymbol{x}) .
$$

The parameter settings we require will turn out to be the following:

$$
\widehat{\lambda} \text { satisfying poly }(T d)^{T} \cdot \hat{\lambda}^{T / 2}=\underline{\lambda} \varepsilon_{\mathrm{PRG}}, \quad \delta_{\text {horz }}:=\frac{1}{K d D},
$$

where $K$ is a suitably large absolute constant and $T>2$ is a constant that will be set later (for now, the most important thing to notice is that since $T>2$, the exponent on $\widehat{\lambda}$ above is strictly greater than 1 ).

The mollifier checks are designed precisely to check that the above properties Inequalities 18 and 19 actually hold at $x$, and thus Theorem 2.2 corresponds to the fact that these properties hold with high probability for a random $x \sim \mathcal{N}(0,1)^{n}$.

With the above intuition in place, we proceed to define the mollifier checks that check for each of the above types of properties (1) and (2).

4.3.1 Checking Local Hyperconcentration. For each $0 \leq i \leq d-1$, MollifierCHecks contains a check corresponding to Inequality 18 . The "inequality" portion of the check is

$$
s_{i+1,0} \leq e \widehat{\lambda} s_{i, 1}
$$

and the "softness" parameter of the check is 1; so for this check CHeck in MollifierChecks, the associated "soft check" is

$$
\operatorname{SoftCheck}_{\text {CHECK }}(x)=\sigma\left(\ln \left(\frac{e \widehat{\lambda} s_{i, 1}}{s_{i+1,0}}\right)\right) \text {. }
$$

We refer to these $d$ elements of MoLLIFIERCHEcks as "anticoncentration checks" or as "diagonal checks."

4.3.2 Checking Insensitivity under Noise. For each pair of values $i \in\{0, \ldots, d\}, j \in\{0, D-2\}$, MollifierCHeCKs contains a pair of checks corresponding to Inequality 19 . The "inequality" portion of the first (respectively, second) check of the pair is

$$
s_{i, j}(x) \geq \exp \left(2 \delta_{\text {horz }}\right) \cdot s_{i, j+1}(x)
$$

(respectively, $\left.\left.s_{i, j+1}(x) \geq \exp \left(2 \delta_{\text {horz }}\right) \cdot s_{i, j}(x)\right)\right)$, and the "softness" parameter of each of these checks is $\delta_{\text {horz }}$. So for these two elements CHeck, CHeck' of MollifierChecks the associated "soft checks" are

$$
\begin{aligned}
& \operatorname{SoftCheck}_{\mathrm{CHECK}}(x)=\sigma\left(\delta_{\text {horz }}^{-1} \ln \left(\frac{\exp \left(2 \delta_{\text {horz }}\right) \cdot s_{i, j}(x)}{s_{i, j+1}(x)}\right)\right) \text { and } \\
& \operatorname{SoftCheck}_{\mathrm{CHECK}^{\prime}}(x)=\sigma\left(\delta_{\text {horz }}^{-1} \ln \left(\frac{\exp \left(2 \delta_{\text {horz }}\right) \cdot s_{i, j+1}}{s_{i, j}}\right)\right) .
\end{aligned}
$$

We refer to these $2 d D$ checks as "noise-insensitivity checks" or as "horizontal checks."

This concludes the definition of MOLLIFIERCHECKs, so recalling Definition 4.2 the definition of Mollifier $p$ is now complete. We turn to proving Theorem 2.2 .

\subsection{Breaking Down the Mollification Error for the Proof of Theorem 2.2}

Recalling the definition of MoLLIfIERCHEcks from Section 4.3, the approach to proving Theorem 2.2 is clear. We will show that each of the local hyperconcentration (diagonal) checks passes "with room to spare" with high probability over $\boldsymbol{x}$, and that likewise each of the noise-insensitivity (horizontal) checks passes with room to spare with high probability over $\boldsymbol{x}$. The two theorems stated below give the desired bounds:

THEOREM 4.11 (Local hyPERCONCENTRATION, ROUGH ANALOgUe of Corollary 10 of [4]). For each $i \in\{0, \ldots, d-1\}$, except with probability at most $\varepsilon_{\mathrm{PRG}} /(8 d)$ over $\boldsymbol{x} \sim \mathcal{N}(0,1)^{n}$, Inequality 18 holds, i.e.

$$
s_{i+1,0}(\boldsymbol{x}) \leq \widehat{\lambda} s_{i, 1}(\boldsymbol{x}) .
$$

Theorem 4.12 (Noise-InSENSITIVITy, ANALOgue of Lemma 11 OF [4]). For each $i \in\{0, \ldots, d\}$ and $j \in\{0, \ldots, D-1\}$, except with probability at most $\varepsilon_{\mathrm{PRG}} /(8(d+1) D)$ over $\boldsymbol{x} \sim \mathcal{N}(0,1)^{n}$, Inequality 19 holds, i.e.

$$
s_{i, j}(\boldsymbol{x}) \approx \delta_{\text {horz }} s_{i, j+1}(\boldsymbol{x}) .
$$

Proof of Theorem 2.2 using Theorem 4.11 and Theorem 4.12.

By a union bound over failure probabilities, we have that with probability at least $1-\varepsilon_{\mathrm{PRG}} / 4$ Inequality 18 holds for all $i$ and Inequality 19 holds for all $i, j$. If Inequality 18 holds for a given $i$ then $\ln \frac{e \widehat{\lambda} s_{i, 1}}{s_{i+1,0}} \geq 1$ and the diagonal check Equation (21) evaluates to 1 . If Inequality 19 holds for a given $i, j$ then $\delta_{\text {horz }}^{-1} \ln \left(\frac{\exp \left(2 \delta_{\text {horz }}\right) \cdot s_{i, j}}{s_{i, j+1}}\right) \geq 1$ and the horizontal check Equation (22) evaluates to 1, and similarly the horizontal check Equation (23) evaluates to 1.

It remains to prove Theorems 4.11 and 4.12 .

\section{PROOF OF THE LOCAL HYPERCONCENTRATION THEOREM}

In this section we present the key new ingredient underlying our main result, the Local Hyperconcentration Theorem for degree- $d$ polynomials (Theorem 4.11). As alluded to in the Introduction, this result says that with high probability over a Gaussian $\boldsymbol{x} \sim \mathcal{N}(0,1)^{n}$, the $\lambda$-zoom of a degree- $d$ polynomial $p$ at $x$ (i.e. the polynomial $p_{\lambda \mid x}$ ) is attenuated - intuitively, it is "very close to a constant polynomial". We refer to this result as a "local hyperconcentration theorem" since by Lemma 3.21 attenuation of $p_{\lambda \mid x}$ implies that the random variable $p_{\lambda \mid x}(\boldsymbol{y})$ (for $\left.\boldsymbol{y} \sim \mathcal{N}(0,1)^{n}\right)$ is hyperconcentrated; this property will play a crucial role in our later technical arguments.

For technical reasons related to the definition of our statistics (essentially because each statistic $s_{i, j}(x)$ is an average of polynomials - recall Section 4.2), the actual statement we will need is one that is about a distribution of polynomials rather than a single polynomial. However, for clarity of exposition we first state the "one-polynomial" version of our local hyperconcentration theorem below:

THEOREM 5.1 (LOCAL HYPERCONCENTRATION THEOREM FOR A SINGLE POLYNOMIAL). Let $g: \mathbb{R}^{n} \rightarrow \mathbb{R}$ be a polynomial of degree at most $d$. Fix parameters $R \geq 1,0<\varepsilon \leq 1,0<\beta<1$, and assume

$$
\lambda \leq \frac{\varepsilon}{R^{2}} \cdot\left(\frac{\beta}{d}\right)^{C \log d}
$$


(where $C$ is a certain universal constant). Then for $x \sim \mathcal{N}(0,1)^{n}$, except with probability at most $\beta$ we have that the randomly zoomed polynomial $g_{\lambda \mid x}$ is $(R, \varepsilon)$-attenuated; i.e.,

$$
\operatorname{HyperVar}_{R}\left[g_{\lambda \mid x}\right] \leq \varepsilon \cdot\left\|g_{\lambda \mid x}\right\|_{2}^{2} .
$$

Another way to phrase the conclusion is that for $\boldsymbol{h}=g_{\lambda \mid \boldsymbol{x}}$, except with probability $\beta$ we have that $\boldsymbol{h}$ is such that

$$
\operatorname{HyperVar}_{R}[\boldsymbol{h}] \leq(d / \beta)^{O(\log d)} \cdot R^{2} \lambda \cdot\|\boldsymbol{h}\|_{2}^{2} .
$$

Notice that the dependencies here on $R$ and $\lambda$ are "correct" in the sense that if one intuitively thinks of $\lambda$ as "infinitesimal", we expect that $\boldsymbol{h}$ will have $\Theta(\lambda)$ weight at level 1, negligible weight above level 1, and the definition of HyperVar ${ }_{R}$ multiplies this $\Theta(\lambda)$ level-1 weight by $R^{2}$. The "error factor" in this result, $(d / \beta) O(\log d)$ with $\beta \sim \varepsilon_{\text {PRG }}$, essentially becomes our final seed length (divided by $\log n)$.

Because of the need to analyze the statistics introduced in Section 4.1.2, we will often need to work with a distribution over polynomials rather than a single polynomial. We therefore introduce the following generalization of Definition 5.4, which captures the notion of a distribution over polynomials being attenuated on average:

Definition 5.2 (Nice distribution of polynomials is attenuated on average). Let $\left(g_{\boldsymbol{v}}\right)_{\boldsymbol{v} \sim \Upsilon}$ be a nice (in the sense of Section 4.2) distribution of polynomials over $\mathbb{R}^{n}$. For $R \geq 1$ and $0<\varepsilon \leq 1$, we say that the distribution $\left(g_{v}\right)_{v \in \Upsilon}$ is $(R, \varepsilon)$-attenuated on average if $\mathbf{E}_{\boldsymbol{v}}\left[\operatorname{HyperVar}_{R}\left[g_{\boldsymbol{v}}\right]\right] \leq \varepsilon \cdot \mathbf{E}_{\boldsymbol{v}}\left[\left\|g_{\boldsymbol{v}}\right\|_{2}^{2}\right]$.

The actual main result we prove in this section is Theorem 5.3, which generalizes Theorem 5.1 to a nice distribution of polynomials:

THEOREM 5.3 (LOCAL HYPERCONCENTRATION THEOREM FOR A NICE DISTRIBUTION OF POLYNOMIALS). Let $\left(g_{v}\right)_{v \sim r}$ be a nice (in the sense of Section 4.2) distribution of degree-d polynomials. Fix parameters $R \geq 1,0<\varepsilon \leq 1,0<\beta<1$, and assume

$$
\lambda \leq \frac{\varepsilon}{R^{2}} \cdot\left(\frac{\beta}{d}\right)^{C \log d}
$$

(where $C$ is a certain universal constant). Then for $\boldsymbol{x} \sim \mathcal{N}(0,1)^{n}$, except with probability at most $\beta$ we have that the distribution $\left(\left(g_{v}\right)_{\lambda \mid x}\right)_{\boldsymbol{v} \in \Upsilon}$ is $(R, \varepsilon)$-attenuated on average; i.e.,

$$
\mathrm{E}_{\boldsymbol{v}}\left[\operatorname{HyperVar}_{R}\left[\left(g_{\boldsymbol{v}}\right)_{\lambda \mid \boldsymbol{x}}\right]\right] \leq \varepsilon \cdot \mathrm{E}_{\boldsymbol{v}}\left[\left\|\left(g_{\boldsymbol{v}}\right)_{\lambda \mid \boldsymbol{x}}\right\|_{2}^{2}\right] .
$$

Theorem 4.11 follows from Theorem 5.3 directly by setting parameters as follows:

Proof of Theorem 4.11 using Theorem 5.3. We instantiate Theorem 5.3 with its nice distribution " $\left(g_{v}\right)_{v \sim r}$ " being $\mathcal{F}_{i, 0}$, its " $R$ " parameter being set to $\underline{R}$ defined in Equation (13), its " $\lambda$ ” parameter being $\underline{\lambda}$, its " $\varepsilon$ " parameter being $\hat{\lambda}$, and its " $\beta$ " parameter being $\varepsilon_{\mathrm{PRG}} /(8 d)$. Recalling Equation (16) and Equation (17) we have

$$
\begin{gathered}
s_{i, 0}(x)=\underset{\boldsymbol{v}}{\mathbf{E}}\left[g_{\boldsymbol{v}}(x)^{2}\right], \quad s_{i, 1}(x)=\mathbf{E}_{\boldsymbol{v}}\left[\left\|\left(g_{\boldsymbol{v}}\right)_{\underline{\lambda} \mid x}\right\|_{2}^{2}\right], \\
\text { and } s_{i+1,0}(x)=\mathbf{E}_{\boldsymbol{v}}\left[\operatorname{HyperVar}_{\underline{R}}\left[\left(g_{\boldsymbol{v}}\right)_{\underline{\lambda}} \mid x\right]\right] .
\end{gathered}
$$

Recalling the settings of $\underline{\lambda}$ and $\hat{\lambda}$ from Equation (4) and Equation (20), we see that the bound required in Equation (24) indeed holds, and so we can apply Theorem 5.3, and its conclusion gives precisely the desired conclusion of Theorem 4.11 .

In the rest of this section we prove Theorem 5.3. We first explain the high-level structure of the argument in Section 5.1 and then give the formal proof in the rest of the section.

\subsection{A Useful Definition, and the High-Level Argument Underlying Theorem 5.3}

Before we can give the high level idea of the proof of Theorem 5.3 we need a refined notion of a polynomial being attenuated:

Definition 5.4 (Attenuated polynomial, refined notion). Let $g$ : $\mathbb{R}^{n} \rightarrow \mathbb{R}$ be a polynomial of degree at most $d$. For $k \geq 0, R \geq 1$, and $0<\varepsilon \leq 1$, we say that the polynomial $g$ is $(k, R, \varepsilon)$-attenuated if

$$
\sum_{|\beta|>k} R^{2|\beta|} \widehat{g}(\beta)^{2}=\operatorname{HyperVar}_{R}\left[g^{>k}\right] \leq \varepsilon \cdot\|g\|_{2}^{2} .
$$

Similarly, if $\left(g_{v}\right)_{\boldsymbol{v} \sim \Upsilon}$ is a nice (in the sense of Section 4.2) distribution of polynomials over $\mathbb{R}^{n}$, we say that the distribution $\left(g_{v}\right)_{v \in \Upsilon}$ is $(k, R, \varepsilon)$-attenuated on average if

$$
\mathbf{E}_{\boldsymbol{v}}\left[\operatorname{HyperVar}_{R}\left[g_{\boldsymbol{v}}^{>k}\right]\right] \leq \varepsilon \cdot \mathbf{E}_{\boldsymbol{v}}\left[\left\|g_{\boldsymbol{v}}\right\|_{2}^{2}\right]
$$

Note that $g$ being $(0, R, \varepsilon)$-attenuated is the same as $g$ being $(R, \varepsilon)$ attenuated as defined earlier (see Definition 3.19), and likewise for $\left(g_{v}\right)_{\boldsymbol{v} \sim \Upsilon}($ see Definition 5.2).

With this refined notion of attenuation in hand we can explain the high level idea of our local hyperconcentration theorem. For ease of exposition, below we sketch the underlying ideas in the "one-polynomial" setting of Theorem 5.1 (the same ideas drive the proof of Theorem 5.3).

So, we are given a degree- $d$ polynomial $g$ and the goal is to argue that with high probability over a random point $x \sim \mathcal{N}(0,1)^{n}$, the polynomial $g_{\lambda \mid x}$ is $(R, \varepsilon)$-attenuated, i.e. $(0, R, \varepsilon)$-attenuated. A simple but crucial insight pointing the way is that random zooms compose: in more detail, if $0<\lambda, \lambda^{\prime}<1$ are two noise rates and $\boldsymbol{x}, \boldsymbol{x}^{\prime}$ are two independent $\mathcal{N}(0,1)^{n}$ random variables, then the distribution of the composed random zoom $\left(g_{\lambda \mid x}\right)_{\lambda^{\prime} \mid x^{\prime}}$ is identical to the distribution of $g_{\lambda \lambda^{\prime} \mid z}$ where $z \sim \mathcal{N}(0,1)^{n}$. With this in mind, it is natural to view a random zoom at the small noise rate $\lambda$ as a "strong" random zoom which is obtained by composing a sequence of $\log d$ many "weaker" random zooms at larger noise rates. ${ }^{5}$ If we can prove that a "weak" random zoom with high probability causes a $(k, R, \varepsilon)$-attenuated polynomial to become $(k / 2, R, \varepsilon)$-attenuated, then since any degree- $d$ polynomial is trivially $(d, R, \varepsilon)$-attenuated, a simple union bound over $\log d$ many applications of this "onestage" result yields the desired random zoom lemma for $g$. This is precisely the high-level structure of our argument; see Theorem 5.8 for a formal statement of the one-stage result in the more general setting of a nice distribution of polynomials.

We proceed to give intuition for the proof of the one-stage result. In this setting we are now given $g$ which is a $(k, R, \varepsilon)$-attenuated polynomial; intuitively this means that the amount of Hermite weight it has at levels $k+1, k+2, \ldots$ is very small compared to the

\footnotetext{
${ }^{5}$ The idea of decomposing a "strong"random zoom into multiple "weak" random zooms is due to Avi Wigderson.
} 
total Hermite weight of $g$ at all levels $0,1, \ldots$ We must argue that with high probability over $\boldsymbol{x} \sim \mathcal{N}(0,1)^{n}$, after a random zoom at $\boldsymbol{x}$ the polynomial $\boldsymbol{q}:=g_{\lambda \mid \boldsymbol{x}}$ is $(k / 2, R, \varepsilon)$-attenuated, i.e. the amount of Hermite weight $\boldsymbol{q}$ has at levels $k / 2+1, k / 2+2, \ldots$ is very small relative to the total Hermite weight of $\boldsymbol{q}$ at levels $0,1, \ldots$ This is naturally done via a two part argument. The first part is to argue two-norm retention: this amounts to showing that with high probability over $\boldsymbol{x}$, the squared two-norm of $\boldsymbol{q}$ does not become too small relative to the squared two-norm of $p$. The argument for this is based on the Carbery-Wright anticoncentration bound (Theorem 3.23) and the tail bound for Gaussian polynomials (Theorem 3.12); see Section 5.2 for a precise statement and proof of this part. The second part is to argue attrition of the high-degree Hermite weight: this amounts to showing that with high probability after a random zoom, the amount of Hermite weight at levels $k / 2+1, k / 2+2, \ldots$ becomes very small relative to the squared two-norm of $p$. The argument for this is based on Corollary 3.6 and Markov's inequality; see Section 5.3 for a precise statement and proof.

\subsection{First Part of the Proof of the One-Stage Local Hyperconcentration Theorem: Retention}

The main result of this section is Lemma 5.6. Its proof uses the following proposition:

Proposition 5.5. Let $\left(g_{v}\right)_{v \sim \Upsilon}$ be a nice distribution of polynomials of degree at most $k$. Then for $\boldsymbol{x} \sim \mathcal{N}(0,1)^{n}$, except with probability at most $\beta^{\prime}$ we have

$$
\underset{\boldsymbol{v}}{\mathrm{E}}\left[\left\|\left(g_{\boldsymbol{v}}\right)_{\lambda \mid x}\right\|_{2}^{2}\right] \geq\left(\frac{\beta^{\prime}}{O(k)}\right)^{2 k} \underset{\boldsymbol{v}}{\mathbf{E}}\left[\left\|g_{\boldsymbol{v}}\right\|_{2}^{2}\right] .
$$

Proof. Let $r(x):=\mathrm{E}_{\boldsymbol{v}}\left[\left\|\left(g_{v}\right)_{\lambda \mid x}\right\|_{2}^{2}\right]$. We observe that $r$ is a nonnegative degree- $2 k$ polynomial with mean

$$
\begin{aligned}
& \mathbf{E}_{\boldsymbol{x} \sim \mathcal{N}(0,1)^{n}}[r(\boldsymbol{x})]=\underset{\boldsymbol{v}}{\mathbf{E}} \underset{\boldsymbol{x} \sim \mathcal{N}(0,1)^{n}}{\mathbf{E}}\left[\left\|\left(g_{\boldsymbol{v}}\right)_{\lambda \mid \boldsymbol{x}}\right\|_{2}^{2}\right] \\
& =\underset{\boldsymbol{v}}{\mathrm{E}} \underset{\boldsymbol{x}, \boldsymbol{x}^{\prime} \sim \mathcal{N}(0,1)^{n}}{\mathrm{E}}\left[g_{\boldsymbol{v}}\left(\sqrt{1-\lambda} \boldsymbol{x}+\sqrt{\lambda} \boldsymbol{x}^{\prime}\right)\right] \\
& =\underset{\boldsymbol{v}}{\mathrm{E}} \underset{\boldsymbol{g} \sim \mathcal{N}(0,1)^{n}}{\mathrm{E}}\left[g_{\boldsymbol{v}}(\boldsymbol{g})^{2}\right]=\underset{\boldsymbol{v}}{\mathrm{E}}\left[\left\|g_{\boldsymbol{v}}\right\|_{2}^{2}\right] .
\end{aligned}
$$

The claimed result now follows immediately from the CarberyWright anticoncentration bound Theorem 3.23 applied to $p=r$, since $\|r\|_{2} \geq\|r\|_{1}=\mathrm{E}[r]=\mathrm{E}_{\boldsymbol{v}}\left[\left\|g_{\boldsymbol{v}}\right\|_{2}^{2}\right]$.

One way to think of the nice distribution of degree- $k$ polynomials in Proposition 5.5 is that it is " $(k,+\infty, 1)$-attenuated on average." Lemma 5.6 relaxes this requirement and shows that a similar result holds for a nice distribution that is $(k, S, 1)$-attenuated on average for a modestly large $S$. We defer the proof of Lemma 5.6 to the full version of this paper.

Lemma 5.6. Let $\left(g_{v}\right)_{v \sim \Upsilon}$ be a nice distribution of polynomials that is $(k, S, 1)$-attenuated on average for some $k \in \mathbb{N}^{+}$. Fix a parameter $0<\beta^{\prime}<1$. Then for $\boldsymbol{x} \sim \mathcal{N}(0,1)^{n}$, except with probability at most $\beta^{\prime}$ we have

$$
\underset{\boldsymbol{v}}{\mathbf{E}}\left[\left\|\left(g_{\boldsymbol{v}}\right)_{\lambda \mid \boldsymbol{x}}\right\|_{2}^{2}\right] \geq\left(\frac{\beta^{\prime}}{O(k)}\right)^{2 k} \underset{\boldsymbol{v}}{\mathbf{E}}\left[\left\|g_{\boldsymbol{v}}\right\|_{2}^{2}\right]
$$

provided that (for a certain universal constant $C$ )

$$
S \geq C k \log \left(3 / \beta^{\prime}\right) / \beta^{\prime} .
$$

\subsection{Second Part of the Proof of the One-Stage Local Hyperconcentration Theorem: Attrition}

The attrition result we establish in this subsection, Lemma 5.7, is a fairly direct consequence of Corollary 3.6. (Note that Lemma 5.7 does not require that the nice distribution $\left(g_{v}\right)_{v \sim r}$ be attenuated on average - it holds for any nice distribution of degree- $d$ polynomials.) We defer the proof of Lemma 5.7 to the full version of this paper.

LEMMA 5.7. Let $\left(g_{v}\right)_{v \sim \Upsilon}$ be a nice distribution of polynomials of degree at most $d$. Fix parameters $0<\beta^{\prime}<1, R^{\prime} \geq 1, m \in \mathbb{N}^{+}$, $0<\varepsilon \leq 1$, let $c>0$ be a sufficiently small constant, and assume $\lambda \leq \frac{c^{4} \varepsilon \beta^{\prime 5}}{R^{\prime 2} m^{3} d}$. Then for $x \sim \mathcal{N}(0,1)^{n}$,

$$
\frac{1}{\varepsilon} \cdot \underset{\boldsymbol{v}}{\mathbf{E}}\left[\operatorname{HyperVar}_{R^{\prime}}\left[\left(\left(g_{\boldsymbol{v}}\right)_{\lambda \mid \boldsymbol{x}}\right)^{\geq m}\right]\right] \leq\left(\frac{O\left(c \beta^{\prime}\right)}{m}\right)^{4 m} \underset{\boldsymbol{v}}{\mathbf{E}}\left[\left\|g_{\boldsymbol{v}}\right\|_{2}^{2}\right]
$$

holds except with probability at most $\beta^{\prime}$.

\subsection{Putting the Pieces Together: Proof of the Local Hyperconcentration Theorem}

Combining Lemma 5.6 and Lemma 5.7 (with the " $k$ " and " $m$ " parameters satisfying $m=\lfloor k / 2\rfloor$, and adjusting constants), we may deduce the following, which is our "one-stage local hyperconcentration theorem:"

THEOREM 5.8 (ONE-STAGE LOCAL HYPERCONCENTRATION THEO$\mathrm{REM})$. Let $\left(g_{v}\right)_{\boldsymbol{v} \sim} \sim$ be a nice distribution of polynomials of degree at most $d$, and assume the distribution is $(k, S, 1)$-attenuated on average for some $1 \leq k \leq d$. Fix parameters $R^{\prime} \geq 1,0<\beta^{\prime}<1,0<\varepsilon^{\prime} \leq 1$, and assume

$$
S \geq C k \log \left(3 / \beta^{\prime}\right) / \beta^{\prime}, \quad \lambda \leq \frac{\varepsilon^{\prime} \beta^{\prime 5}}{C R^{\prime 2} k^{3} d}
$$

for a suitably large universal constant $C$. Then except with probability at most $\beta^{\prime}$ over $\boldsymbol{x} \sim \mathcal{N}(0,1)^{n}$, the distribution $\left(\left(g_{\boldsymbol{v}}\right)_{\lambda \mid \boldsymbol{x}}\right)_{\boldsymbol{v} \sim \Upsilon}$ is $\left(\lfloor k / 2\rfloor, R^{\prime}, \varepsilon^{\prime}\right)$-attenuated on average.

Note that a nice distribution of degree- $d$ polynomials is $(d, S, 1)$ attenuated on average for any $S$. We can take $S=C d \log \left(3 / \beta^{\prime}\right) / \beta^{\prime}$ and perform a first application of Theorem 5.8 on $\left(g_{v}\right)_{v \sim \Upsilon}$ with its $R^{\prime}$ parameter set to $S$ and its $\varepsilon^{\prime}$ parameter set to 1 , and infer that except with failure probability at most $\beta^{\prime}$ the distribution $\left(\left(g_{v}\right)_{\lambda \mid z}\right)_{v \sim \Upsilon}$ is $(\lfloor d / 2\rfloor, S, 1)$-attenuated on average. Repeating this a total of $\lceil\log d\rceil$ times, with each repetition having its $\beta^{\prime}$ parameter set to $\beta /(\lceil\log d\rceil+1)$, its $R^{\prime}$ parameter set to $S=C d \log \left(3 / \beta^{\prime}\right) / \beta^{\prime}$ (for simplicity), and its $\varepsilon^{\prime}$ parameter set to 1 , we get that except with probability $\beta \cdot\left(1-\frac{1}{\lceil\log d\rceil+1}\right)$, the distribution $\left(\left(g_{v}\right)_{\lambda^{\prime} \mid z}\right)_{v \in \Upsilon}$ is $(1, S, 1)$-attenuated on average, where $\lambda^{\prime}=\left(\frac{\beta^{6}}{C^{2} d^{6} \log ^{2}\left(3 / \beta^{\prime}\right)}\right)^{\lceil\log d\rceil}$. Finally, we perform one last application of Theorem 5.8 with its $k$ parameter set to 1 , its $\lambda^{\prime}$ parameter set to $\underline{\lambda}$, its $\varepsilon^{\prime}$ parameter set to the " $\varepsilon$ " of Theorem 5.3 , and its $\beta^{\prime}$ parameter set to $\beta /(\lceil\log d\rceil+1)$ and its $R^{\prime}$ parameter set to $R$. We get the conclusion of Theorem 5.3 
as stated at the beginning of this section, and the proof of the local hyperconcentration theorem is complete.

Due to space constraints the remainder of this paper is deferred to the full version.

\section{ACKNOWLEDGMENTS}

We thank Avi Wigderson for a key conceptual suggestion toward the proof of the Local Hyperconcentration theorem.

\section{REFERENCES}

[1] Anthony Carbery and James Wright. 2001. Distributional and $L^{q}$ norm inequalities for polynomials over convex bodies in $\mathbb{R}^{n}$. Mathematical Research Letters 8 , 3 (2001), 233-248.

[2] Ilias Diakonikolas, Daniel Kane, and Jelani Nelson. 2010. Bounded independence fools degree-2 threshold functions. In Proceedings of the 51st Annual Symposium on Foundations of Computer Science (FOCS). 11-20.

[3] Daniel Kane. 2011. $k$-Independent Gaussians Fool Polynomial Threshold Functions. In Proceedings of the 26th Conference on Computational Complexity (CCC). 252-261.
[4] Daniel Kane. 2011. A Small PRG for Polynomial Threshold Functions of Gaussians. In Proceedings of the 52nd Annual Symposium on Foundations of Computer Science (FOCS). 257-266.

[5] Daniel Kane. 2012. A structure theorem for poorly anticoncentrated Gaussian chaoses and applications to the study of polynomial threshold functions. In Proceedings of the 53rd Annual Symposium on Foundations of Computer Science (FOCS). 91-100.

[6] Daniel Kane. 2014. A pseudorandom generator for polynomial threshold functions of Gaussians with subpolynomial seed length. In Proceedings of the 29th Annual Conference on Computational Complexity (CCC). 217-228.

[7] Daniel Kane. 2015. A polylogarithmic PRG for degree 2 threshold functions in the Gaussian setting. In Proceedings of the 30th Conference on Computational Complexity (CCC). 567-581.

[8] Pravesh Kothari and Raghu Meka. 2015. Almost Optimal Pseudorandom Generators for Spherical Caps. In Proceedings of the 47th Annual on Symposium on Theory of Computing (STOC). 247-256.

[9] Raghu Meka and David Zuckerman. 2010. Pseudorandom generators for polynomial threshold functions. In Proceedings of the 42nd ACM Symposium on Theory of Computing (STOC). 427-436.

[10] Raghu Meka and David Zuckerman. 2013. Pseudorandom generators for polynomial threshold functions. SIAM F. Comput. 42, 3 (2013), 1275-1301.

[11] Ryan O'Donnell. 2014. Analysis of Boolean Functions. Cambridge University Press. Available at http://analysisofbooleanfunctions.net/. 